\title{
Inventario florístico del Cerro El Cebú y zonas aDyacentes en la Reserva de la Biosfera El Triunfo (Polígono V), Chiapas, MÉxico
}

\author{
Jorge Martínez-Meléndez ${ }^{1,3}$, Miguel Ángel Pérez-Farrera ${ }^{1}$ y Oscar Farrera-Sarmiento ${ }^{2}$ \\ Herbario Eizi Matuda, Escuela de Biología, Universidad de Ciencias y Artes de Chiapas. \\ Libramiento Norte Poniente s/n, Col. Lajas Maciel, Tuxtla Gutiérrez, Chiapas, México. \\ ${ }^{2}$ Departamento de Flora, Instituto de Historia Natural y Ecología. \\ Calzada de los Hombres Ilustres s/n, Tuxtla Gutiérrez, Chiapas, México. \\ ${ }^{3}$ Autor para la correspondencia. Correo-e: red_falcon10@yahoo.com.mx
}

\begin{abstract}
Resumen: Se registró un total de 502 especies pertenecientes a 301 géneros y 115 familias en el Cerro El Cebú y zonas adyacentes del Polígono V de la Reserva de la Biosfera El Triunfo, Chiapas, México. Las familias con mayor riqueza fueron Orchidaceae, Fabaceae, Asteraceae, Bromeliaceae y Polypodiaceae, con 58, 29, 28, 17 y 17 especies, respectivamente. Se reconocieron cinco tipos de comunidades vegetales, de los cuales el bosque mesófilo de montaña fue la más diversa, con 234 especies, seguido del bosque de coníferas con 150 y el bosque tropical perennifolio con 127. El bosque de encino y el bosque tropical caducifolio incluyeron únicamente 71 y 39 especies, respectivamente. Del total de taxa para el área, solamente trece están incluidos en listas de especies en peligro de extinción.

Palabras clave: bosque de coníferas, bosque mesófilo de montaña, bosque tropical perennifolio, florística, reserva de la biosfera.

Abstract: A total of 502 species that belong to 115 families and 301 genera were recorded for Mt. El Cebu and adjacent zones in Polygon V of the El Triunfo Biosphere Reserve. The most speciose families were Orchidaceae, Fabaceae, Asteraceae, Bromeliaceae and Polypodiaceae with 58,29, 28, 17, and 17 species, respectively. Six plant communities were recognized in the area, among which the cloud forest was the most diverse with 234 species, followed by pine-oak forest with 150 species, and the tropical rain forest with 127. The oak forest and the tropical deciduous forest were less diverse, with 71 and 39 species, respectively. Only 13 species are included in the Mexican red list of endangered taxa.
\end{abstract}

Keywords: cloud forest, floristics, pine-oak forest, tropical forest, Biosphere reserve.

$\mathbf{E}_{\mathrm{n}}^{1}$ trópico húmedo alberga la mayor biodiversidad del mundo, sin embargo, en dicha región se localiza la mayoría de los países poco desarrollados, cuya población humana ejerce una presión fuerte sobre los recursos naturales (Wilson, 1992). Por ejemplo, México, Brasil, Colombia e Indonesia se encuentran entre los países de más alta diversidad (ConABIo-INE, 1994). La alta biodiversidad presente en México ha sido explicada a partir de factores como su posición geográfica entre las zonas Neártica y Neotropical, así como su heterogeneidad ambiental (Rzedowski, 1978). Se estima que el número de especies de angiospermas en México asciende a 24,500 (Espejo-Serna et al. 2004), y el de helechos y afines a un poco más de 1,000 especies (Mickel y Smith, 2004). Por lo tanto, aún existe la necesidad de elaborar inventarios biológicos en las áreas con mayor di- versidad en el país, pues se calcula que existe únicamente información para menos de $15 \%$ de las especies (CONABIOINE, 1994). Por otro lado, México ha perdido más de $95 \%$ de sus bosques tropicales húmedos (selvas altas perennifolias y bosques mesófilos de montaña), más de la mitad de sus bosques templados y un porcentaje difícil de cuantificar de sus zonas áridas, pero que sin duda rebasa la mitad de su superficie original (Challenger, 1998). Chiapas es el segundo estado del país en términos de riqueza florística (RamírezMarcial et al., 1998), con aproximadamente 8,250 especies (Breedlove, 1981). Sin embargo, el conocimiento de su flora es aún incompleto. Más aún, su riqueza florística está fuertemente amenazada: March-Mifsut y Flamenco (1996) mencionan que la deforestación en Chiapas entre 19761991 fue de $1.9 \%$ anual; de seguir así, esto significa que en 
51 años podría desaparecer la cobertura boscosa del estado.

Se han realizado diversos estudios florísticos para Chiapas, muchos de ellos en la Sierra Madre (Matuda, 1950a, 1950b; Miranda, 1952, 1957; Breedlove, 1981; Long y Heath, 1991; Williams-Linera, 1991; Bachem y Rojas, 1994; Castillo, 1996), pero para las zonas norte y sur los estudios florísticos son escasos (Martínez et al., 1994; GutiérrezBáez, 2004). Por otro lado, Ochoa-Gaona (1996) realizó un estudio de la vegetación de la selva El Ocote en la zona centro, y Ramírez-Marcial et al. (1998) llevaron a cabo un análisis florístico y sucesional para el cerro Huitepec, en la meseta central del estado.

El objetivo de este trabajo fue elaborar el inventario florístico del Cerro El Cebú y zonas adyacentes en el Polígono $\mathrm{V}$ de la Reserva de la Biosfera El Triunfo, Chiapas, caracterizando las asociaciones vegetales presentes.

\section{Área de estudio}

El Cerro El Cebú se localiza aproximadamente a $60 \mathrm{~km}$ al suroeste de la ciudad de Independencia, en el municipio de La Concordia. Se ubica entre las coordenadas $15^{\circ} 49^{\prime} y$ $15^{\circ} 50^{\prime} \mathrm{N}$, y $93^{\circ} 02^{\prime}$ y $93^{\circ} 05^{\prime} \mathrm{O}$, en la porción sureste de la zona núcleo V de la Reserva de la Biosfera El Triunfo (REBITRI) (figura 1). Colinda al norte con las fincas Arroyo Negro y Nueva Linda, al sur con la finca Santa Cruz, al este con las fincas El Plan y Las Nubes y al oeste con el rancho El Vergel. $\mathrm{Su}$ altitud oscila entre 900 y 2,250 m s.n.m. El clima de la zona es Aw2(w)igw, es decir, cálido subhúmedo con lluvias en verano (García, 1987), con temperatura media anual mayor de $22^{\circ} \mathrm{C}$ y temperatura del mes más frío mayor que $18^{\circ} \mathrm{C}$
(INE-SEMARNAP, 1999). De acuerdo con Müllerried (1957), pertenece a la provincia fisiográfica Tierras Altas de Chiapas-Guatemala, específicamente a la subprovincia Sierra de Chiapas; el mismo autor menciona que en la mayor parte del área de la reserva predominan rocas graníticas y metamórficas del Paleozoico y calizas-areniscas del Paleozoico superior, y que los suelos de las cumbres y vertientes poseen suelos complejos de montaña (cafés y podzólicos). La REBITRI está enclavada en el parteaguas de la Sierra Madre de Chiapas, donde se encuentra la frontera entre dos regiones hidrográficas del estado, la de Grijalva-Usumacinta (vertiente de la Depresión central), y la de la Costa (vertiente del Pacífico) (INE-SEMARnAP, 1999).

\section{Métodos}

Se realizaron colectas de ejemplares botánicos en el período 2004-2005 siguiendo el método propuesto por Long y Heath (1991). Se recolectaron al menos cuatro duplicados por muestra. El primer ejemplar siempre se depositó en el Herbario Eizi Matuda (HEM) y los duplicados fueron destinados a los herbarios CAS, CHIP, MEXU, MO y XAL. Los especímenes se determinaron utilizando claves de identificación de diversas floras y trabajos florísticos para el neotrópico (Breedlove, 1981, 1995; Daniel, 1999; Standley y Williams, 1975; Sosa y Gómez-Pompa 1978-2005; Davidse et al., 1994, 1995; Gentry, 1993). Para reconocer a las especies en riesgo se consultó la Norma Oficial Mexicana (NOM-059-ECOL-2001; Semarnat, 2001) y la Lista Roja de Especies Amenazadas de la Unión Internacional para la Conservación de la Naturaleza (IUCN, 2007).

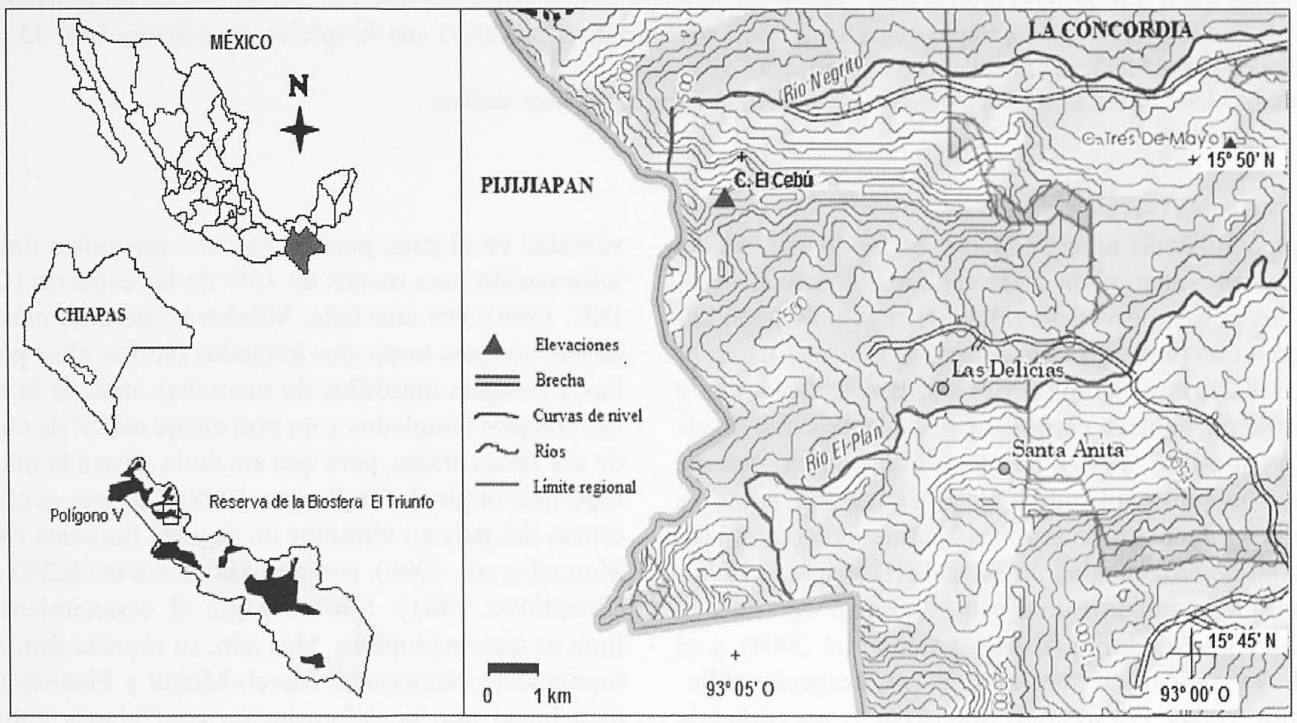

Figura 1. Localización geográfica del Cerro El Cebú, Polígono V, Reserva de la Biosfera El Triunfo, Chiapas, México. 


\section{Resultados}

Riqueza. Se recolectaron 656 números de colecta en el periodo 2004-2005 en el Cerro El Cebú y zonas adyacentes (apéndice 1). Asimismo, durante la revisión del herbario HEM permitió registrar 140 ejemplares adicionales, haciendo un total de 796. De esta manera se obtuvo un listado florístico que incluye 502 especies pertenecientes a 301 géneros y 115 familias (cuadro 1). Las Magnoliophyta o angiospermas son el grupo mejor representado, con 293 especies de dicotiledóneas y con 138 de monocotiledóneas. Las Pteridophyta o helechos y afines incluyeron 68 taxa, mientras que de las Gimnospermas únicamente se encontraron tres especies. La familia con mayor número de especies fue Orchidaceae con 58, seguida de Fabaceae con 29, Asteraceae con 28, y Polypodiaceae y Bromeliaceae con 17 (figura 2). La familia más rica en géneros fue Orchidaceae con 33, seguida por Asteraceae con 22, Fabaceae con 18 y Rubiaceae con 10 (figura 2). Los géneros con mayor riqueza son Tillandsia con 12; Polypodium, Peperomia y Epidendrum con nueve, y Quercus con ocho especies. Respecto a las formas biológicas, las hierbas fueron predominantes con 327 especies, seguidas por los arbustos con 71 , los árboles con 70, las lianas o bejucos con 35 y los arborescentes con ocho. La mayoría de las especies fueron terrestres (377), mientras que las epífitas fueron 95.

Tipos de vegetación. Se detectaron cinco tipos de vegetación de acuerdo con la clasificación de Rzedowski (1978). De éstos, el bosque mesófilo de montaña fue el más diverso con 234 especies, seguido del bosque de coníferas con 150 y el bosque tropical perennifolio con 127 especies. Los tipos de vegetación con menor riqueza de especies fueron el bosque de encino y el bosque tropical subcaducifolio, con 71 y 39 especies, respectivamente.
Cuadro 1. Riqueza de los grupos de plantas recolectadas en el Cerro El Cebú y zonas adyacentes, Polígono V, Reserva de la Biosfera El Triunfo, Chiapas, México.

\begin{tabular}{lccc}
\hline Grupo & Familias & Géneros & Especies \\
\hline Pteridophyta & 16 & 32 & 68 \\
Gimnospermae & 3 & 3 & 3 \\
Angiospermae & & & \\
$\quad \begin{array}{l}\text { monocotiledóneas } \\
\text { dicotiledóneas }\end{array}$ & 18 & 75 & 138 \\
Total & 78 & 191 & 293 \\
\hline
\end{tabular}

Bosque tropical perennifolio. Este tipo de vegetación se encuentra en altitudes de 1,325 a 1,857 m s.n.m., en los alrededores de la finca Santa Cruz y hacia el lado oeste del rancho Buenavista III. Presenta tres estratos. El más alto es el arbóreo, con plantas de $20 \mathrm{~m}$ o más de altura, con especies como Ficus cookii, Alibertia edulis, Clusia flava y Ulmus mexicana, así como Eugenia amatenanguensis, E. acapulcensis y E. capuli. El estrato arbustivo incluye plantas de 1.5 a 4 $\mathrm{m}$ de altura, destacando Palicourea padifolia, Ardisia compressa, Parathesis chiapensis, Hoffmannia psychotriifolia, helechos arborescentes como Cyathea fulva y palmas como Chamaedorea quezalteca, C. pinnatifrons, C. nubium, Geonoma oxycarpa y $G$. seleri. En el estrato herbáceo dominan especies de hasta $1 \mathrm{~m}$ de altura como Maianthemum paniculatum, Spigelia scabra, Campyloneurum tenuipes, Phytolacca icosandra y P. purpurascens. Entre las especies epífitas destacan Anthurium chiapasense, Mostera siltepecana, $M$. acacoyaguensis, Macrolinium sp., Peperomia collocata y Tillandsia butzii.

Bosque tropical subcaducifolio. Esta comunidad se extiende entre 950 a 1,550 m s.n.m., en la parte de un sendero cono-

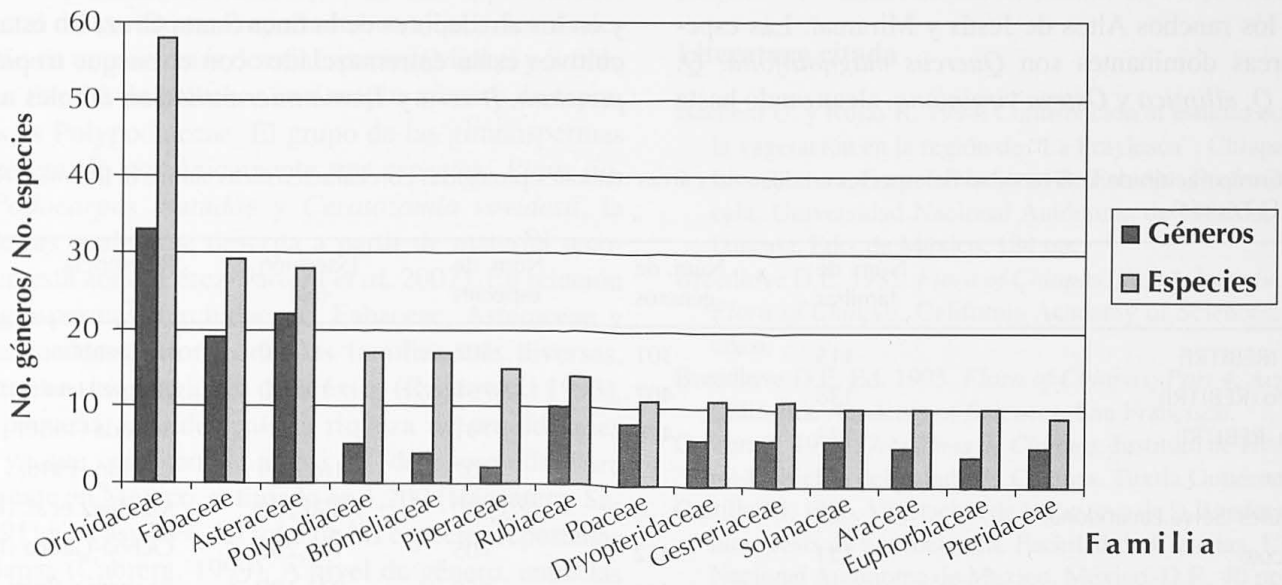

Figura 2. Riqueza de familias, géneros y especies encontradas en el Cerro El Cebú y zonas adyacentes en la Reserva de la Biosfera El Triunfo y Chiapas. 
cido como Cinco Caminos, que comunica el rancho Buenavista III con la finca Santa Cruz. En ella se distinguen tres estratos. El estrato arbóreo está formado por especies como Quercus elliptica, Solanum ochraceo-ferrugineum e Inga punctata, las cuales no rebasan los $9 \mathrm{~m}$ de altura. Entre los arbustos destacan especies de no más de $3 \mathrm{~m}$ de altura como Moninna xalapensis y Piper schiedeanum, mientras que en el estrato herbáceo dominan Heliconia latisphata, Melinis minutiflora, Lasiacis grisebachii y Maianthemum paniculatum. El número de especies epífitas es menor, destacando Anthurium chiapasense, A. cerrobaulense, Maxillaria densa, Lepanthes sp. y Tillandsia ponderosa.

Bosque de coníferas. Se encuentra entre 936 y $1,590 \mathrm{~m}$ s.n.m., y abarca la mayor parte de la zona de estudio, es decir, desde la finca Las Nubes hasta incluir los ranchos Morelia, Corazón de Jesús, Altos de Jesús y parte de las fincas Arroyo Negro y Nueva Linda. El estrato arbóreo incluye especies de Pinus y Quercus que alcanzan alturas de 12-20 m. El estrato arbustivo está dominado por algunas especies de 1 a 2 m de altura como Acalypha leptopoda, Ceratozamia vovidesii y Calliandra houstoniana, mientras que en el herbáceo se presentan con mayor frecuencia especies de hasta $0.8 \mathrm{~m}$ de altura como Chamaecrista rufa, Crotalaria bupleurifolia, Cypripedium irapeanum y algunas especies rastreras como Ipomoea tiliacea, I. silvicola e I. hastigera. En pequeñas áreas a altitudes de 1,000 a 1,800 m s.n.m. se encontraron asociaciones de Pinus oocarpa con Quercus acutifolia, $Q$. magnoliifolia y Liquidambar styraciflua. Las epífitas son abundantes, principalmente sobre los encinos; como ejemplos de ellas se puede citar a Mormodes nagelii, Stelis bidentata, Peperomia collocata, Isochilus carnosiflorus, Brassia verrucosa, Tillandsia seleriana y $T$. juncea.

Bosque de encino. Se observaron fragmentos de encinares entre 1,161 a 1,470 m s.n.m., en las cercanías de finca Las Nubes, y los ranchos Altos de Jesús y Miramar. Las especies arbóreas dominantes son Quercus magnoliifolia, $Q$. castanea, $Q$. elliptica y Ostrya virginiana, alcanzando hasta
$12 \mathrm{~m}$ de altura. Los arbustos más frecuentes son Calliandra houstoniana, C. grandiflora, Malvaviscus arboreus y Cordia spinescens, alcanzando hasta $2 \mathrm{~m}$ de altura, mientras que las hierbas (0.1-0.3 m de altura) están representadas por Achimenes longiflora, Cuphea aequipetala, Lasiacis grisebachii y Oplismenus setarius. Entre las epífitas destacan Prosthechea ochracea, P. cochleata, Monstera siltepecana, Maxillaria variabilis, Tillandsia usneoides y Meiracyllium trinasutum.

Bosque mesófilo de montaña. En la cima del Cerro El Cebú se localiza un bosque mesófilo de montaña a altitudes entre 2,230 y 2,246 m s.n.m. Aquí dominan especies de los géneros Quercus, Vaccinium y Cavendishia en el estrato arbóreo, alcanzando únicamente hasta $3 \mathrm{~m}$ de altura; en el estrato arbustivo (1 a $2.5 \mathrm{~m}$ de altura) se encuentran Moussonia elegans, Lippia substrigosa y Coriaria ruscifolia. Entre las especies del estrato herbáceo se pueden mencionar a representantes de Werauhia, Tillandsia, Beschorneria, Agave, Epidendrum, Encyclia, Russelia, Polypodium y Phlebodium Las epífitas más notables son Scaphyglottis livida, Epidendrum cerinum y Dichaea glauca. En altitudes de 2,200 a 2,230 m s.n.m., correspondientes a las partes más altas del Cerro El Cebú, se desarrolla una asociación con una composición florística diferente, con especies árboreas tales como Quercus sp., Cavendishia crassifolia y Pinus oocarpa. Entre los arbustos están Senecio cobanensis, mientras que las hierbas están representadas por Maianthemum paniculatum, Hydrocotyle mexicana y Commelina diffusa, y algunas gramíneas como Melinis minutiflora y Panicum sp. Las epífitas son muy abundantes; entre las más importantes se encuentran Tillandsia ponderosa, T. guatemalensis, T. usneoides, Stelis cobanensis, Epidendrum laucheanum, Pleopeltis angusta, Polypodium loriceum y Elaphoglossum peltatum.

En el área también existen cultivos, principalmente cafetales, sobre todo en el noroeste del rancho Buenavista III y en los alrededores de la finca Santa Cruz. En esta área los cultivos están entremezclados con el bosque tropical. Inga punctata, I. vera y Trema micrantha son árboles utilizados

Cuadro 2. Comparación de la diversidad de especies en diferentes áreas naturales protegidas con características similares (ReBıTRı = Reserva de la Biosfera El Triunfo).

\begin{tabular}{|c|c|c|c|c|c|}
\hline Localidad & $\begin{array}{l}\text { Núm. de } \\
\text { familias }\end{array}$ & $\begin{array}{l}\text { Núm. de } \\
\text { géneros }\end{array}$ & $\begin{array}{l}\text { Núm. de } \\
\text { especies }\end{array}$ & $\begin{array}{c}\text { Extensión } \\
\text { (ha) }\end{array}$ & Referencia \\
\hline C. El Cebú (REBITRI) & 115 & 301 & 502 & 450 & Este estudio \\
\hline C. El Triunfo (REBITRI) & 138 & 407 & 751 & 10,000 & Long y Heath (1991) \\
\hline C. Ovando (REBITRI) & 122 & 476 & 791 & $\cdots$ & Matuda (1950) \\
\hline La Sepultura & 72 & -- & 407 & 167,309 & Castillo (1996) \\
\hline Montes Azules (Selva Lacandona) & 61 & $\cdots$ & 3,400 & $1,550,200$ & Martínez et al. (1994) \\
\hline Selva El Ocote & 121 & 452 & 705 & 101,288 & Ochoa-Gaona (1996) \\
\hline La Fraylesca & 129 & 441 & 858 & 831,180 & Bachem y Rojas (1994) \\
\hline Los Tuxtlas, Veracruz & 214 & -- & 3,000 & 155,000 & Ruiz y Durán, (2004) \\
\hline
\end{tabular}


para proporcionar sombra a las plantaciones del café. En ocasiones se encuentran especies herbáceas como Oplismenus setarius, Sida acuta, Tradescantia zanonia y Verbena litoralis. Las epífitas son escasas en estos cultivos, a pesar de lo cual fueron encontradas Peperomia galioides, Polypodium lindenianum, $P$. furfuraceum y Vittaria graminifolia.

\section{Discusión}

Nuestro inventario incluye 502 especies en el Cerro El Cebú, cifra que representa $6.08 \%$ del total de la flora de Chiapas, de acuerdo con la cifra estimada para el estado de 8,250 especies (Breedlove, 1981). Actualmente, en la base de datos del herbario HEM se tiene un registro total de 2,468 especies para toda la reserva del Triunfo (R. Martínez-Camilo, com. pers.), lo que significa que el Cerro El Cebú incluye a $20.34 \%$ de la flora de la reserva.

El cuadro 2 compara la diversidad florística del Cerro El Cebú con otras áreas tropicales de Chiapas y Veracruz. Del cuadro se desprende que la diversidad del Cebú es elevada, ya que en una superficie de tan sólo 450 hectáreas se alcanza una diversidad de más de 500 especies. Otras dos áreas con una alta diversidad florística, la reserva de Montes Azules en Chiapas y la de Los Tuxtlas en Veracruz, albergan un poco más de 3,000 especies, pero sus superficies son considerablemente mayores.

Del total de especies del inventario, trece están enlistadas en el apéndice 1 de la Norma Oficial Mexicana NOM-059ECOL-2001 (Semarnat, 2002). Adicionalmente, se encontraron cinco especies en la Lista Roja de la IUCN (2007): Pinus oocarpa, Podocarpus matudae, Saurauia scabrida, Oreopanax sanderianus y Liquidambar styraciflua (apéndice 1).

En la flora del Cerro El Cebú y áreas adyacentes es notable el elevado número de especies de helechos y plantas de grupos afines; en conjunto corresponden a un poco más de $5 \%$ del total de los taxones reportados en el país, los cuales se calculan en un poco más de mil especies (Mickel y Smith, 2004). La familia de helechos con mayor número de representantes es Polypodiaceae. El grupo de las gimnospermas está representado por únicamente tres especies: Pinus oocarpa, Podocarpus matudae y Ceratozamia vovidesii, la última de las cuales fue descrita a partir de material recolectado en esta zona (Pérez-Farrera et al. 2007). En relación a las angiospermas, Orchidaceae, Fabaceae, Asteraceae y Bromeliaceae destacaron como las familias más diversas, al igual que en otras regiones de México (Rzedowski 1993). En esta pequeña área del país la riqueza de orquídeas es notable, ya que corresponde a casi $5 \%$ de la orquideoflora total presente en México, estimado en 1,200 (Hágsater y Salazar, 1991) y a casi $10 \%$ del total de las especies reportadas para Chiapas (Cabrera, 1999). A nivel de género, entre las angiospermas los géneros más diversos fueron Tillandsia, Polypodium, Peperomia, Epidendrum y Quercus, los cuales se encuentran entre los géneros con mayor número de especies para todo México (Villaseñor, 2004). Asimismo, se encontró que el bosque mesófilo de montaña es el tipo de vegetación más diverso, seguido del bosque de coníferas y el bosque tropical perennifolio. Esto concuerda con otros estudios de bosque mesófilo en el país, que reportan una alta diversidad (Luna y Espinosa, 1993).

El inventario florístico realizado en El Cerro el Cebú y zonas adyacentes enfatiza la importancia de llevar a cabo estudios en zonas de muy alta biodiversidad, ya que en una superficie tan pequeña se registraron más de 500 especies de plantas vasculares.

\section{Agradecimientos}

El primer autor agradece a Chris Davidson y Sharon Christoph la beca de tesis otorgada dentro del proyecto IFT05 "Inventario Florístico en la Reserva de la Biosfera El Triunfo, Chiapas, México". El segundo autor agradece al progama PROMEP los fondos otorgados (103.5/07/2363). Agradecemos la determinación de material a W.D. Stevens (Asclepiadaceae), F. Lorea (Lauraceae), A. Espejo (Bromeliaceae, Liliaceae), A. Smith, J. Mickel, María E. LópezMolina (Pteridophyta), J.F. Smith (Gesneriaceae), A. Bornstein (Piperaceae), T. Croat (Araceae), H. Quero-Rico, R. Martínez-Camilo (Arecaceae), R. Liesner (varias familias), C. Taylor (Rubiaceae), J. Pruski (Asteraceae), M. Sousa, R. Cruz-Durán (Fabaceae), P. Carrillo (Crassulaceae), J. Ricketson (Myrsinaceae), R. Ortíz-Gentry (Menispermaceae), M. Martínez-Gordillo (Euphorbiaceaee, Lamiaceae), G. Davidse (Poaceae, Cyperaceae), R. Solano, Carlos R. Beutelspacher, N. Martínez-Meléndez (Orchidaceae) y H. Gómez-Domínguez (Ericaceae). A A.P. Vovides agradecemos por la revisión del resumen en inglés y a J.A. Meave y dos revisores anónimos por los comentarios y sugerencias que permitieron mejorar el manuscrito.

\section{Literatura citada}

Bachem U. y Rojas R. 1994. Contribución al estudio ecológico de la vegetación en la región de "La Fraylesca", Chiapas. Tesis de Licenciatura, Escuela Nacional de Estudios Profesionales Iztacala, Universidad Nacional Autónoma de México, Los Reyes Iztacala, Edo. de México, 184 pp.

Breedlove D.E. 1981. Flora of Chiapas. Part I. Introduction to the Flora of Chiapas. California Academy of Sciences, San Francisco.

Breedlove D.E. Ed. 1995. Flora of Chiapas. Part 4, Acanthaceae. California Academy of Sciences, San Francisco.

Cabrera T. 1999. Orquídeas de Chiapas. Instituto de Historia Natural-Gobierno del Estado de Chiapas. Tuxtla Gutiérrez, Chis.

Castillo J.J. 1996. Vegetación de la Reserva de la Biosfera La Sepultura. Tesis de Licenciatura, Facultad de Ciencias, Universidad Nacional Autónoma de México, México, D.F., 40 pp.

Challenger A. 1998. Utilización y Conservación de los Ecosistemas Terrestres de México: Pasado, Presente y Futuro. Comisión 
Nacional para el Conocimiento y Uso de la Biodiversidad, Instituto de Biología, UNAM y Agrupación Sierra Madre, S.C., México, D.F.

ConABIo-INE (Comisión Nacional para el Conocimiento y Uso de la Biodiversidad-Instituto Nacional de Ecología). 1994. Programa Piloto de Inventarios Biológicos en Áreas Naturales Protegidas. México, D.F.

Daniel T.F. Ed. 1999. Flora of Chiapas. Part 5. Compositae-Heliantheae. California Academy of Sciencies, San Francisco.

Davidse G., Sousa-Sánchez M. y Charter A.O. Eds. 1994. Flora Mesoamericana. Vol. 6. Alismataceae a Cyperaceae. Universidad Nacional Autónoma de México, Missouri Botanical Garden y The Natural History Museum (London), México, D.F.

Davidse G., Sousa-Sánchez M. y Knapp S. 1995. Flora Mesoamericana. Vol. 1. Psilotaceae a Salviniaceae. Universidad Nacional Autónoma de México, Missouri Botanical Garden y The Natural History Museum (London), México, D.F.

Espejo-Serna A., López-Ferrari A.R. y Salgado-Ugarte I. 2004. A current estimate of angiosperm diversity in Mexico. Taxon 53:127-130.

García E. 1987. Modificaciones al Sistema de Clasificación Climática de Köppen (para Adaptarlo a las Condiciones de la República Mexicana). Universidad Nacional Autónoma de México, México D.F.

Gentry A.H. 1993. A Field Guide to the Families and Genera of Woody Plants of Northwest South America (Colombia, Ecuador, Peru), with Supplementary Notes on Herbaceous Taxa. University of Chicago Press, Chicago.

Gutiérrez C. 2004. Listado florístico del Norte de Chiapas: Catazajá y límites con Palenque. Polibotánica 17:107-124.

Hágsater E. y Salazar V. Eds. 1991. Orquídeas de México: Icones Orchidacearum. Vol. 1. Asociación Mexicana de Orquideología A.C., México D.F.

INE-SEmarnap [Instituto Nacional de Ecología-Secretaría de Medio Ambiente, Recursos Naturales y Pesca]. 1999. Programa de Manejo, Reserva de la Biosfera de La Sepultura. México, D.F. 249 pp.

IUCN. 2007. IUCN Red List of Threatened Species. <www.iucnredlist.org>

Long A. y Heath M. 1991. Flora of the El Triunfo Biosphere Reserve, Chiapas, Mexico: A preliminary floristic inventory and the plant communities of Polygon I. Anales del Instituto de Biología, Universidad Nacional Autónoma de México. Serie Botánica 62:133-172.

Luna I., Almeida L. y Llorente J. 1989. Reconocimiento florístico y consideraciones fitogeográficas del bosque mesófilo de montaña de las Cañadas de Ocuilán, estados de Morelos y México. Anales del Instituto de Biología. Universidad Nacional Autónoma de México. Serie Botánica 59:63-87.

March-Mifsut I.J. y Flamenco S.A. 1996. Evaluación rápida de la deforestación en las Áreas Naturales Protegidas de Chiapas (1970-1993). El Colegio de la Frontera Sur, San Cristóbal de las Casas, Chiapas, México.

Martínez E., Ramos C. y Chiang F. 1994. Lista florística de la Lacandona, Chiapas. Boletín de la Sociedad Botánica de México 54:99-177.

Matuda E. 1950a. A contribution to our knowledge of wild flora of Mt. Ovando. The American Midland Naturalist 43:195-223.

Matuda E. 1950b. A contribution to our knowledge of the wild and cultivated flora of Chiapas. I. Districts Soconusco and Mariscal.
The American Midland Naturalist 44:513-616.

Mickel J.T. y Smith A.R. 2004. The Pteridophytes of Mexico. Memoirs of The New York Botanical Garden 88:1-1054.

Miranda F. 1952. La Vegetación de Chiapas. 2 Vols. Gobierno del Estado de Chiapas, Tuxtla Gutiérrez, Chis.

Miranda F. 1957. Vegetación de la Vertiente del Pacífico de la Sierra Madre de Chiapas (México) y sus Relaciones Florísticas. Instituto Botánico de Chiapas, Tuxtla Gutiérrez, Chis. [Reproducido en López-Sánchez C. Comp. 1993. Lecturas Chiapanecas, Vol. 6, pp. 79-101, Gobierno del Estado de Chiapas, México, D.F.]

Müllerried F.K.G. 1957. Geología de Chiapas. Gobierno del Estado de Chiapas, México, D.F.

Ochoa-Gaona S. 1996. La vegetación de la Reserva El Ocote a lo largo del cañón del río La Venta. En: Vásquez-Sánchez M.A y March-Mifsut I.J. Eds. Conservación y Desarrollo Sustentable en la Selva El Ocote, Chiapas, pp. 45-61. El Colegio de la Frontera Sur, Centro de Estudios para la Conservación de los Recursos Naturales, A.C. y Comisión Nacional para el Conocimiento y Uso de la Biodiversidad, San Cristóbal de Las Casas.

Pérez-Farrera M.A., González-Astorga J., Avendaño S. e Iglesias C.G. 2007. A new species of Ceratozamia (Zamiaceae) from the Sierra Madre of Chiapas, Mexico, with comments on species relationships. Botanical Journal of the Linnean Society 153:393-400.

Ramírez-Marcial N., Ochoa-Gaona S. y González-Espinosa M. 1998. Análisis florístico y sucesional en la Estación Biológica Cerro Huitepec, Chiapas, México. Acta Botanica Mexicana 44:59-85.

Rzedowski J. 1978. Vegetación de México. Limusa, México, D.F.

Rzedowski J. 1993. Diversidad y orígenes de la flora fanerogámica de México. En Ramamoorthy T.P., Bye R., Lot A. y Fa J. Comps. Diversidad Biológica de México: Orígenes y Distribución, pp. 129-145, Universidad Nacional Autónoma de México, México, D.F.

Semarnat [Secretaría del Medio Ambiente y Recursos Naturales]. 2002. Norma Oficial Mexicana NOM-059-ECOL-2001, Protección ambiental-Especies nativas de México de flora y fauna silvestres-Categorías de riesgo y especificaciones para su inclusión, exclusión o cambio-Lista de especies en riesgo. Diario Oficial de la Federación. 2a Sección, 6 de marzo de 2002.

Sosa V. y Gómez-Pompa A. Eds. 1978-2005. Flora de Veracruz. (Fascículos varios). Instituto de Ecología, A.C. y Universidad de California, Riverside, Xalapa.

Standley P.C. y Williams L.O. 1961-1975. Flora of Guatemala. Fieldiana: Botany 24.

Villaseñor J.L. 2004. Los géneros de plantas vasculares de la Flora de México. Boletín de la Sociedad Botánica de México 75:105-135.

Williams Linera G. 1991. Nota sobre la estructura del estrato arbóreo del bosque mesófilo de montaña en los alrededores del campamento "El Triunfo", Chiapas. Acta Botanica Mexicana 13:1-7.

Wilson E.O. 1992. The effects of complex social-life on evolution and biodiversity. Oikos 63:13-18.

Recibido: 26 de septiembre de 2007

Aceptado: 17 de abril de 2008 
Apéndice 1. Listado de especies del Cerro El Cebú y zonas adyacentes en el Polígono V de la Reserva de La Biosfera El Triunfo, Chiapas, México. Relación de Colectores: Jorge Martínez Meléndez (JMM), Miguel Ángel Pérez Farrera (MAPF), Nayely Martínez Meléndez (NMM), María Evangelina López Molina (MELM), Angélica Ríos Alegría (ARA), César Andrés Pérez Bonifaz (CAPB), Fanny Evangelina Pérez Castillo (FEPC), Jeremías López Chagala (JLC), Francisco Hernández Najarro (FHN). Categoría de riesgo NOM-059-ECOL-2001: (A) Amenazada, (Pr) Protección especial. Categoría de riesgo IUCN 2007: Vulnerable (VU, vulnerable), Near Threatened (NT, casi amenazado), Lower Risk ( $L R$, bajo riego). Forma de Crecimiento ( $F C$ ): $\mathrm{Hi}=\mathrm{Hierba}, \mathrm{Ar}=\mathrm{Arbusto}, \mathrm{Ab}=$ Arborescente, $\mathrm{A}=$ Árbol, $\mathrm{Be}=$ bejuco o liana. Tipo de Hábito $(\mathrm{TH})$ : Ep= Epífita, He= Hemiepífita, $\mathrm{T}=\mathrm{Terrestre}, \mathrm{Tr}=\mathrm{Trepadora}, \mathrm{R}=$ Rupícola, $\mathrm{S}=$ Saprófita, $\mathrm{P}=$ Parásita. Fenología (EF): $\mathrm{Fl}=\mathrm{Flor}, \mathrm{Fr}=$ Fruto, Fe= Fértil (helechos). Mes de Colecta $(\mathrm{MC}): 01=\mathrm{Enero}$ hasta $12=$ Diciembre. El guión (-) significa que no se tiene información del ejemplar. Símbolos: $\left(^{*}\right)$ Nueva especie, $(+)$ nuevos registros para El Triunfo.

\begin{tabular}{llllll}
\hline Especies y sus datos de colecta & FC & TH & EF & MC \\
\hline
\end{tabular}

\section{PTERIDOPHYTA}

\section{LYCOPHYTA}

Lycopodiaceae

Lycopodium clavatum L. JMM985

$\begin{array}{cccc}\mathrm{Hi} & \mathrm{Te} & \mathrm{Fe} & 06 \\ \mathrm{Hi} & \mathrm{Te} & - & 08\end{array}$

\section{Selaginellaceae}

Selaginella pallescens (C.Presl) Spring JMM1108

\section{MONILOPHYTA}

\section{Aspleniaceae}

Asplenium achilleifolium (M.Martens et Galeotti) Liebm. MELM175

Asplenium cuspidatum Lam. JMM978

Asplenium hoffmannii Hieron. FEPC158

Asplenium miradorense Liebm. MELM187, 704, 705

Asplenium radicans L. MELM186

Asplenium serra Langsd. et Fisch. MELM188, 197

Hi

\section{Athyriaceae}

Diplazium franconis Liebm. MELM179

Diplazium werckleanum H.Christ. MELM170, 200

\section{Blechnaceae}

Blechnum glandulosum Kaulf. ex Link MELM163, 172

Blechnum occidentale L. ARA263

Blechnum polypodioides Raddi JMM552

\section{Cyatheaceae}

Cyathea fulva (M.Martens et Galeotti) Fée MELM192, 204 (Pr)

\section{Dryopteridaceae}

Arachniodes denticulata (Sw.) Ching FEPC124, MELM176

Athyrium palmense (H.Christ) Lellinger JMM537

Elaphoglossum aff. guatemalense (Klotzsch) T.Moore JMM1160

Elaphoglossum latifolium (Sw.) J.Sm. FEPC121

Elaphoglossum muscosum (Sw.) T.Moore JMM1153

Elaphoglossum peltatum (Sw.) Urb. JMM1152, MELM178

Elaphoglossum piloselloides (C.Presl) T.Moore JMM1023

Elaphoglossum sp. JMM993 Hi

Nephrolepis pectinata (Willd.) Schott. JMM536

Phanerophlebia juglandifolia (Humb. et Bonpl. ex Willd.) J.Sm. JMM783,

MELM174, 184, 202

Polystichum hartwegii (Klotzsch) Hieron. MELM189, 190, 706

Tectaria transiens (C.V.Morton) A.R.Sm. MELM174a, 701

\section{Equisetaceae}

Equisetum hyemale L. ARA262

Equisetum hyemale var. affine (Engelm.) A.A.Eaton JMM1020

$\begin{array}{llcl}\mathrm{Hi} & \mathrm{Te} & - & 02 \\ \mathrm{Hi} & \mathrm{Ep} & \mathrm{Fe} & 0 \\ \mathrm{Hi} & \mathrm{Ep} & - & 0 \\ \mathrm{Hi} & \mathrm{Te} & - & 02 \\ \mathrm{Hi} & \mathrm{Te} & - & 02 \\ \mathrm{Hi} & \mathrm{Ep} & - & 0\end{array}$

$\mathrm{Hi} \quad \mathrm{Te} \quad \mathrm{Fe} \quad 02$

$\begin{array}{lll}\mathrm{Hi} & \mathrm{Te} & -\end{array}$

$\begin{array}{llll}\mathrm{Hi} & \mathrm{Te} & - & 0 \\ \mathrm{Hi} & \mathrm{Te} & \mathrm{Fe} & 0 \\ \mathrm{Hi} & \mathrm{Te} & \mathrm{Fe} & 11\end{array}$

$\mathrm{Ab}$

$\mathrm{Hi}$

$\mathrm{Hi}$

$\mathrm{Hi}$

$\mathrm{Hi}$

$\mathrm{Hi}$

$\mathrm{Hi}$

$\mathrm{Hi}$

Ep

$\mathrm{Hi}$

$\mathrm{Hi}$

$\mathrm{Hi}$

$\mathrm{Hi}$

$\mathrm{Hi}$

$\mathrm{Hi}$

02

06

03

02

02 


\begin{tabular}{l} 
Especies y sus datos de colecta \\
\hline Gleicheniaceae
\end{tabular}

Gleicheniaceae

Sticherus bifidus (Willd.) Ching JMM975

\section{FC}

TH

$\mathrm{Hi}$

Te

$\mathrm{Fe}$

06

\section{Hymenophyllaceae}

Hymenophyllum lanatum Fée FEPC157

Hymenophyllum polyanthos (Sw.) Sw. MELM194

Trichomanes radicans Sw. FEPC157a, 500, MELM185

Trichomanes reptans Sw. MELM193, 199

\section{Marattiaceae}

Marattia weinmanniifolia Liebm. MELM191

\section{Ophioglossaceae}

Botrychium virginianum (L.) Sw. JMM1068

Ophioglossum reticulatum L. JMM994

\section{Polypodiaceae}

Campyloneurum tenuipes Maxon JMM1004, 1064, JLC49, MELM171

Pecluma alfredii (Rosenst.) M.G.Price JMM1088

Phlebodium areolatum (Humb. et Bonpl. ex Willd.) J. Sm. JMM564, 880, CAPB56

Phlebodium pseudoaureum (Cav.) Lellinger JMM628

Pleopeltis angusta Humb. et Bonpl. ex Willd. JMM1087, JLC57, MELM195

Pleopeltis astrolepis (Liebm.) E.Fourn. JMM547

Pleopeltis macrocarpa var. interjecta (Weath.) A.R.Sm. JMM699

Pleopeltis polylepis (Roem. ex Kunze) T.Moore JMM1163

Polypodium alansmithii R.C.Moran JMM1165

Polypodium cf. remotum Desv. MELM196

Polypodium furfuraceum Schltdl. et Cham. JMM549, 903, 1132

Polypodium lindenianum Kunze JMM933

Polypodium longepinnulatum E.Fourn. JMM1167, JLC34, MELM181

Polypodium loriceum L. JMM698, 1156, FEPC132, MELM183, 702

Polypodium plesiosorum Kunze FEPC173, MELM201

Polypodium sanctae-rosae (Maxon) C.Chr. JMM1140

Polypodium thyssanolepis A.Braun ex Klotzsch JMM1032

\section{Pteridaceae}

Adiantum concinnum Humb. et Bonpl. ex Willd. JMM633, 1092, MELM162

Adiantum pulverulentum L. MELM167

Adiantum wilesianum Hook. MELM168

Cheilanthes aff. cuneata Kaulf. ex Link JMM554

Cheilanthes angustifolia Kunth JMM548

Pityrogramma calomelanos (L.) Link JMM1136

Pteris muricella Fée MELM180Hi

Pteris quadriaurita Retz. MELM165, 173

Vittaria graminifolia Kaulf. JMM575, 1077, FEPC116, MELM177, 182, 198

\section{Schizaeaceae}

Anemia hirsuta (L.) Sw. JMM533

Anemia pasticanaria Moritz ex Prantl. JMM523, 1135

Anemia phyllitidis (L.) Sw. JMM662, 1007

Anemia semihirsuta Mickel MELM164

$\begin{array}{cc}\text { Te } & \mathrm{Fe} \\ \mathrm{Ep} & \mathrm{Fe} \\ \mathrm{Ep}, \mathrm{Te} & \mathrm{Fe} \\ \mathrm{Te} & \mathrm{Fe} \\ \mathrm{Ep}, \mathrm{Te} & \mathrm{Fe} \\ \mathrm{Ep} & \mathrm{Fe} \\ \mathrm{Ep} & \mathrm{Fe} \\ \mathrm{Ep} & \mathrm{Fe} \\ \mathrm{Ru} & \mathrm{Fe} \\ \mathrm{Ep} & - \\ \text { Ep } & \mathrm{Fe} \\ \mathrm{Ep} & \mathrm{Fe} \\ \mathrm{Ep}, \mathrm{Ru} & \mathrm{Fe} \\ \mathrm{Ep} & \mathrm{Fe} \\ \mathrm{Ep} & - \\ \mathrm{Ru} & \mathrm{Fe} \\ \mathrm{Ru} & \mathrm{Fe} \\ \end{array}$

02, 07, 08

\section{Thelypteridaceae}

Thelypteris blanda (Fée) C.F.Reed MELM169, 615

Thelypteris kunthii (Desv.) C.V.Morton JMM932

Thelypteris puberula (Baker) C.V.Morton var. puberula MELM166

$\begin{array}{llcl}\mathrm{Hi} & \mathrm{Te} & - & 02 \\ \mathrm{Hi} & \mathrm{Te} & \mathrm{Fe} & 06 \\ \mathrm{Hi} & \mathrm{Te} & - & 02\end{array}$




\begin{tabular}{lllll}
\hline Especies $y$ sus datos de colecta & FC & TH & EF & MC \\
\hline SPERMATOPHYTA & & & &
\end{tabular}

\section{GIMNOSPERMAE}

\section{Pinaceae}

Pinus oocarpa Schiede ex Schltdl. JMM635 (LR)

A $\mathrm{Te} \quad \mathrm{Fr}$

$\mathrm{Fr}$

\section{Podocarpaceae}

Podocarpus matudae Lundell FEPC149 (NT)

A

Te

03

\section{Zamiaceae}

*Ceratozamia vovidesii Pérez-Farrera, González-Astorga, Avendaño et Iglesias JMM636, 675, 822, CAPB79

\section{ANGIOSPERMAE \\ LILIOPSIDA \\ Amaryllidaceae}

Agave walleriana Baker JMM1036

Beschorneria albiflora Matuda JMM816 (Pr)

Bomarea hirtella (Kunth) Herb. JMM1029, 1089, 1120, JLC68

Furcraea guatemalensis Trel. JMM1040

Ab Te $\quad \mathrm{Fr} \quad 07,11,12$

\section{Araceae}

Anthurium andicola Liebm. FEPC128

+Anthurium cerrobaulense Matuda JMM690, 810

Anthurium chiapasense Standl. JMM686, 915, 916, 945, CAPB78, JLC33,

ARA286, FEPC137

Anthurium titanium Standl. et Steyerm. JMM687

Monstera acacoyaguensis Matuda CAPB89

Monstera siltepecana Matuda JMM901, 971, 1063, EPC115

Philodendron advena Schott FEPC114, 144

Philodendron tripartitum (Jacq.) Schott ARA281, FEPC113

Spathiphyllum matudae G.S.Bunting JMM963, FEPC143

Syngonium podophyllum Schott FEPC112

$\begin{array}{cccc}\mathrm{Hi} & \mathrm{Ru} & - & 07 \\ \mathrm{Hi} & \mathrm{Ru} & \mathrm{Fl}, \mathrm{Fr} & 04 \\ \mathrm{Hi} & \mathrm{Te} & \mathrm{Fl} & 07,08 \\ \mathrm{Hi} & \mathrm{Te} & \mathrm{Fl} & 07 \\ & & & \\ \mathrm{Hi} & \mathrm{Ep} & - & 02 \\ \mathrm{Hi} & \mathrm{Te}, \mathrm{Ep} & \mathrm{Fl} & 04,12 \\ \mathrm{Hi} & \mathrm{Ep}, \mathrm{Te} & \mathrm{Fl}, \mathrm{Fr} & 02,04,06,07,12\end{array}$

\section{Arecaceae}

Chamaedorea nubium Standl. et Steyerm. JMM785, 949, MAPF1822, 1824 (A)

Chamaedorea pinnatifrons (Jacq.) Oerst. CAPB52, JLC53 (A)

Chamaedorea quezalteca Standl. et Steyerm. JMM671, 672, 673, 707, 1062 (A)

Chamaedorea whitelockiana Hodel et N.W.Uhl JMM599, MAPF1798, 1878 (A)

Geonoma oxycarpa Mart. MAPF1825 (A)

Geonoma seleri Burret JMM798

$\begin{array}{cc}\mathrm{Hi} & \mathrm{Te} \\ \mathrm{Hi} & \mathrm{He} \\ \mathrm{Be} & \mathrm{Tr} \\ \mathrm{Be} & \mathrm{Tr} \\ \mathrm{Hi} & \mathrm{Te} \\ \mathrm{Hi} & \mathrm{Te} \\ \mathrm{Be} & \mathrm{Tr}\end{array}$

F

\section{Bromeliaceae}

Catopsis nutans (Sw.) Griseb. JMM1016

Catopsis sessiliflora (Ruiz et Pav.) Mez JMM1100

Pitcairnia imbricata (Brongn.) Regel JMM641

Pitcairnia sp. CAPB70

Tillandsia butzii Mez FEPC140

Tillandsia flabellata Baker JLC38, JMM1022

Tillandsia guatemalensis L.B.Smith. JMM637, 709, FEPC118

Tillandsia juncea (Ruiz et Pav.) Poir. FEPC111, 164

Tillandsia lautneri Ehlers. ARA289

Tillandsia ponderosa L.B.Sm. JMM821, FEPC139 (A)

Tillandsia punctulata Schltdl. et Cham. FEPC123

Tillandsia seleriana Mez JMM805 (A)

Tillandsia tricolor Schltdl. et Cham. ARA297, FEPC165 (A)

Tillandsia usneoides (L.) L. JMM918, 1101, FEPC134

$\begin{array}{ll}\mathrm{Ab} & \mathrm{Te} \\ \mathrm{Ab} & \mathrm{Te} \\ \mathrm{Ab} & \mathrm{Te} \\ \mathrm{Ab} & \mathrm{Te} \\ \mathrm{Ab} & \mathrm{Te} \\ \mathrm{Ab} & \mathrm{Te}\end{array}$

$\begin{array}{cc}\mathrm{Fl}, \mathrm{Fr} & 02,04,06 \\ \mathrm{Fr} & 07 \\ \mathrm{Fl}, \mathrm{Fr} & 08,12 \\ \mathrm{Fr} & 02,11 \\ - & 02 \\ \mathrm{Fl}, \mathrm{Fr} & 04\end{array}$

$\begin{array}{llcc}\mathrm{Hi} & \mathrm{Ep} & \mathrm{FI} & 07 \\ \mathrm{Hi} & \mathrm{Ep} & \mathrm{FI} & 08 \\ \mathrm{Hi} & \mathrm{Te} & \mathrm{FI} & 11 \\ \mathrm{Hi} & \mathrm{Ru} & \mathrm{FI} & 07 \\ \mathrm{Hi} & \mathrm{Ep} & - & 02 \\ \mathrm{Hi} & \mathrm{Ep} & \mathrm{FI} & 07 \\ \mathrm{Hi} & \mathrm{Ep} & \mathrm{FI}, \mathrm{Fr} & 02,11,12 \\ \mathrm{Hi} & \mathrm{Ep} & - & 02 \\ \mathrm{Hi} & \mathrm{Te} & \mathrm{Fr} & 04 \\ \mathrm{Hi} & \mathrm{Ep} & \mathrm{FI} & 02,04 \\ \mathrm{Hi} & \mathrm{Ep} & \mathrm{FI} & 02 \\ \mathrm{Hi} & \mathrm{Ep} & \mathrm{FI} & 04 \\ \mathrm{Hi} & \mathrm{Ep} & - & 03 \\ \mathrm{Hi} & \mathrm{Ep} & \mathrm{Fr} & 02,06,08\end{array}$




Especies y sus datos de colecta

Tillandsia vicentina Standl. JMM818

Tillandsia viridiflora (Beer) Baker FEPC129

Werauhia gladioliflora (H.Wendl.) J.R.Grant JMM815

$\begin{array}{cccc}\text { FC } & \text { TH } & \text { EF } & \text { M } \\ \mathrm{Hi} & \mathrm{Ru} & \mathrm{Fl} & 0 \\ \mathrm{Hi} & \mathrm{Ep} & - & 0 \\ \mathrm{Hi} & \mathrm{Te} & \mathrm{Fl} & 0\end{array}$

\section{Cannaceae}

Canna indica L. JMM1019

$\mathrm{Hi} \quad \mathrm{Te} \quad \mathrm{Fl}, \mathrm{Fr} \quad 07$

\section{Commelinaceae}

Commelina diffusa Burm. f. JMM1069, 1099, ARA295

Commelina erecta L. JMM1161

Tradescantia deficiens Brandegee MAPF1812

Tradescantia zanonia (L.) Sw. JMM565, 794, 1005, JLC36

Tradescantia zebrina Heynh. JMM684

Tradescantia zebrina Heynh. var. mollipila D.R.Hunt. JMM967

Tripogandra serrulata (Vahl) Handlos JMM883, 1013, 1112

$\begin{array}{cccc}\mathrm{Hi} & \mathrm{Te} & \mathrm{Fl} & 08 \\ \mathrm{Hi} & \mathrm{Te} & \mathrm{Fl} & 08 \\ \mathrm{Hi} & \mathrm{Te} & - & 02 \\ \mathrm{Hi} & \mathrm{Te} & \mathrm{Fl}, \mathrm{Fr} & 04,07,11 \\ \mathrm{Hi} & \mathrm{Ru} & \mathrm{FI} & 12 \\ \mathrm{Hi} & \mathrm{Te} & \mathrm{FI} & 06\end{array}$

\section{Cyperaceae}

Calyptrocarya glomerulata (Brongn.) Urb. JMM889

Carex jamesonii Boott MAPF1816

Cyperus hermaphroditus (Jacq.) Standl. JMM561, 886, 931

Cyperus polystachyos Rottb. JMM1006, 1075

$\mathrm{Hi}$ Te

\section{Dioscoreaceae}

Dioscorea floribunda M.Martens et Galeotti JMM1125

Dioscorea matagalpensis Uline JMM595, 660

$\begin{array}{ll}\mathrm{Hi} & \mathrm{Te} \\ \mathrm{Hi} & \mathrm{Te} \\ \mathrm{Hi} & \mathrm{Te} \\ \mathrm{Hi} & \mathrm{Te}\end{array}$

\section{6}

02

FI 06,11

FI 07,08

\section{Haemodoraceae}

Xiphidium caeruleum Aubl. JMM526

Be $\quad \mathrm{Tr}$

$\mathrm{Fr}$

08

$\mathrm{Be}$

$\mathrm{Fl}$

11,12

\section{Heliconiaceae}

Heliconia adflexa (Griggs) Standl. MAPF1787

Heliconia latispatha Benth. JMM651, 797, ARA271

Heliconia schiedeana Klotzsch. JMM1123

\section{Iridaceae}

Orthrosanthus chimboracensis (Kunth) Baker JMM1144

$\begin{array}{ll}\mathrm{Hi} & \mathrm{Te} \\ \mathrm{Hi} & \mathrm{Te} \\ \mathrm{Hi} & \mathrm{Te}\end{array}$

\section{Liliaceae}

Echeandia macrocarpa Greenm. JMM591, 1071

Maianthemum flexuosum (Bertol.) La Frankie MAPF1806

Maianthemum paniculatum (M.Martens et Galeotti) La Frankie JMM670, 696, 908, 942

Maianthemum scilloideum (M.Martens et Galeotti) La Frankie JMM786

\section{Marantaceae}

Calathea coccinea Standl. et Steyerm. JMM896, 951, ARA274

Calathea macrosepala K.Schum. JMM642

Thalia geniculata L. JMM1041

\section{Orchidaceae}

Arpophyllum giganteum Hartw. ex Lindl. FEPC122, 502

Bletia purpurata A.Rich. et Galeotti JMM689, 1090

Bletia purpurea (Lam.) DC. JMM711

Bletia tenuifolia Ames et C.Schweinf. JMM625

Brassia verrucosa Lindl. JMM924

Cattleya skineri Bateman NMM606, FEPC146 (A)

Coelia macrostachya Lindl. JMM708, JLC52

$\begin{array}{cccc}\mathrm{Hi} & \mathrm{Ep} & \mathrm{Fl} & 02 \\ \mathrm{Hi} & \mathrm{Te}, \mathrm{Ru} & \mathrm{FI} & 08,12 \\ \mathrm{Hi} & \mathrm{Te} & \mathrm{FI} & 12 \\ \mathrm{Hi} & \mathrm{Te} & \mathrm{FI} & 11 \\ \mathrm{Hi} & \mathrm{Ep} & \mathrm{FI} & 06 \\ \mathrm{Hi} & \mathrm{Ep} & \mathrm{FI} & 02,12 \\ \mathrm{Hi} & \mathrm{Ep} & \mathrm{Fl}, \mathrm{Fr} & 07,12\end{array}$




\section{Especies y sus datos de colecta}

Cypripedium irapeanum La Llave et Lex. JLC31 (A)

Deiregyne hemichrea (Lindl.) Schltr. FEPC110

Dichaea glauca (Sw.) Lindl. JMM808

Dichaea suaveolens Kraenzl. JMM809, FEPC117

Elleanthus cynarocephalus (Rchb.f.) Rchb.f. JLC51, FEPC125

Encyclia varicosa (Bateman ex Lindl.) Schltr. JMM676, 813

Epidendrum anceps Jacq. JLC60

Epidendrum cardiophorum Schltr. CAPB68

Epidendrum cerinum Schltr. JMM807

Epidendrum ciliare L. JMM1038

Epidendrum laucheanum Rolfe JMM1150

Epidendrum martinezii L. Sánchez et Carnevali JMM992

Epidendrum michuacanum La Llave et Lex. JMM800

Epidendrum myrianthum Lindl. ARA302

Epidendrum racemosum E.A.Noronha JLC48

Goodyera striata Rchb.f. JMM1155

Govenia mutica Rchb.f. CAPB84

Habenaria distans Griseb. JMM1059

Habenaria limosa (Lindl.) Hemsl. JMM514

Habenaria trifida Kunth JMM1109

Isochilus carnosiflorus Lindl. JMM820, 923, JLC55

Lepanthes sp. JMM897

Lockchartia oerstedii Rchb.f. JMM919

Lycaste cruenta Lindl. JMM982

Macrolinium sp. CAPB65

Malaxis sp. JLC50

Maxillaria densa Lindl. JMM646

Maxillaria meleagris Lindl. JMM812

Maxillaria variabilis Bateman ex Lindl. JMM617, 984, ARA264

Meiracyllium trinasutum Rchb.f. JMM1039

Mormodes nagelii L.O.Williams JMM893

Oncidium sphacelatum Lindl. JMM710

Pleurothallis cardiothallis Rchb.f. JMM1043, 1157

Pleurothallis dolichopus Schltr. FEPC147

Pleurothallis matudana C.Schweinf. FEPC126

Pleurothallis sp. JMM1151

Polystachya cerea Lindl. ARA288

Prosthechea baculus (Rchb. F.) W.E.Higgins. JMM1073

Prosthechea ochracea (Lindl.) W.E.Higgins. JMM612, 966, 983,CAPB61

Prosthechea radiata (Lindl.) W.E.Higgins. JMM1037

Rhynchostele rossii (Lindl.) Soto Arenas et Salazar FEPC127

Scaphyglottis crurigera (Bateman ex Lindl.) Ames et Correll ARA273

Scaphyglottis hondurensis (Ames) L.O.Williams JMM804

Scaphyglottis livida (Lindl.) Schltr. JMM644

Scaphyglottis sp. JMM680

Sobralia macrantha Lindl. JMM920, 987, 1042

Spiranthes sp. JMM560

Stelis bidentata Schltr. JMM1003, 921

Stelis cobanensis (Schltr.) Pridgeon et M.W.Chase JMM682

Stelis sp. JMM1154

Triphora mexicana (S.Watson) Schltr. JMM1096

\section{Poaceae}

Chusquea sulcata Swallen MAPF1793

Eleusine indica (L.) Gaertn. JMM885, 1072

Lasiacis grisebachii (Nash) Hitchc. JMM661

Lasiacis nigra Davidse JMM1066

$\begin{array}{llll}\text { FC } & \text { TH } & \text { EF } & \text { MC } \\ \mathrm{Hi} & \mathrm{Te} & \mathrm{FI} & 07 \\ \mathrm{Hi} & \mathrm{Ep} & - & 02 \\ \mathrm{Hi} & \mathrm{Ep} & \mathrm{FI} & 04 \\ \mathrm{Hi} & \mathrm{Ep} & \mathrm{FI} & 04 \\ \mathrm{Hi} & \mathrm{Ep} & \mathrm{FI} & 07 \\ \mathrm{Hi} & \mathrm{Ru} & \mathrm{FI} & 12 \\ \mathrm{Hi} & \mathrm{Ep} & \mathrm{FI} & 07 \\ \mathrm{Hi} & \mathrm{Ep} & \mathrm{FI} & 07 \\ \mathrm{Hi} & \mathrm{Ep} & \mathrm{FI} & 04 \\ \mathrm{Hi} & \mathrm{Ep} & \mathrm{FI} & 07 \\ \mathrm{Hi} & \mathrm{Ep} & \mathrm{FI} & 08 \\ \mathrm{Hi} & \mathrm{Ep} & \mathrm{FI} & 07 \\ \mathrm{Hi} & \mathrm{Te} & \mathrm{FI} & 04 \\ \mathrm{Hi} & \mathrm{Ru} & \mathrm{FI} & 04 \\ \mathrm{Hi} & \mathrm{Ep} & \mathrm{FI} & 07 \\ \mathrm{Hi} & \mathrm{Te} & \mathrm{FI} & 08 \\ \mathrm{Hi} & \mathrm{Te} & \mathrm{FI} & 07 \\ \mathrm{Hi} & \mathrm{Te} & \mathrm{FI} & 08 \\ \mathrm{Hi} & \mathrm{Te} & \mathrm{FI} & 10 \\ \mathrm{Hi} & \mathrm{Te} & \mathrm{FI} & 08 \\ \mathrm{Hi} & \mathrm{Ep} & \mathrm{FI} & 04\end{array}$

04, 06, 07

06

06

06

07

07

12

04

$04,06,11$

07

06

12

07, 08

02

02

08

04

08

$06,07,11$

07

02

04

04

12

12

06

11

06,07

12

08

08

$\begin{array}{llll}\mathrm{Hi} & \mathrm{Ep} & \mathrm{Fl} & 08 \\ \mathrm{Hi} & \mathrm{Te} & \mathrm{FI} & 08\end{array}$

$\begin{array}{cccc}\mathrm{Hi} & \mathrm{Te} & - & 02 \\ \mathrm{Hi} & \mathrm{Te} & \mathrm{FI} & 06,08 \\ \mathrm{Hi} & \mathrm{Te} & \mathrm{Fr} & 12 \\ \mathrm{Hi} & \mathrm{Te} & \mathrm{FI} & 08\end{array}$




Especies y sus datos de colecta

Lasiacis procerrima (Hack.) Hitchc. JMM615, 1127

Lasiacis sp. ARA261

Melinis minutiflora P.Beauv. JMM647, 692

Oplismenus setarius (Lam.) Roem. et Schult. JMM539, 925, 1118

Panicum sp. ARA270, 298

Rhipidocladum racemiflorum (Steud.) McClure ARA282

Setaria parviflora (Poir.) Kerguélen JMM876

\section{Smilacaceae}

Smilax domingensis Willd. JMM974

Smilax mollis Humb. et Bonpl. ex Willd. JMM912

Smilax ornata Lem. JMM668

\section{Typhaceae}

Typha angustifolia L. JMM796

\section{Zingiberaceae}

Costus pictus D.Don. JMM960, CAPB53, ARA284

Costus ruber C.Wright ex Griseb. JMM674

Renealmia mexicana Klotzsch ex Petersen JLC39, CAPB54

\section{MAGNOLIOPSIDA}

\section{Acanthaceae}

Barleria oenotheroides Dum.Cours. JMM529

Tetramerium nervosum Nees JMM940

\section{Actinidaceae}

Saurauia madrensis B.T. Keller et Breedlove JMM618

Saurauia scabrida Hemsl. MAPF1800

\section{Amaranthaceae}

Iresine diffusa Humb. et Bonpl. ex Willd. JMM543, 666, 1002

\section{Anacardiaceae}

Mosquitoxylum jamaicense Krug et Urb. JMM702

Spondias mombin L. JLC46

\section{Annonaceae}

Annona lutescens Saff. CAPB72

\section{Apiaceae}

Daucus montanus Humb. et Bonpl. ex Spreng. JMM904

Hydrocotyle mexicana Schltdl. et Cham. JMM1149, JLC76, ARA268

\section{Apocynaceae}

Mandevilla tubiflora (M.Martens et Galeotti) Woodson JMM944

\section{Aquifoliaceae}

Ilex belizensis Lundell JMM956

\section{Araliaceae}

Oreopanax sanderianus Hemsl. JLC42 (VU)

Oreopanax xalapensis (Kunth) Decne. et Planch. MAPF1791

\section{Asclepiadaceae}

Asclepias curassavica L. JMM930

Blepharodon mucronatum (Schltdl.) Decne. JMM1851

$\begin{array}{lll}\text { FC } & \text { TH } & \\ \mathrm{Hi} & \mathrm{Te} & \mathrm{F} \\ \mathrm{Hi} & \mathrm{Te} & \mathrm{F} \\ \mathrm{Hi} & \mathrm{Te} & \mathrm{F} \\ \mathrm{Hi} & \mathrm{Te} & \mathrm{F} \\ \mathrm{Hi} & \mathrm{Te} & \mathrm{F} \\ \mathrm{Hi} & \mathrm{Te} & \mathrm{F} \\ \mathrm{Hi} & \mathrm{Te} & \mathrm{F}\end{array}$

EF

$\mathrm{Fr}$

$\mathrm{FI}$

$\mathrm{Fl}$

$\mathrm{Fl}$

$\mathrm{Fl}$

$\mathrm{Hi} \quad \mathrm{Te}$

Be $\quad \operatorname{Tr}$

Be $\quad \mathrm{Tr}$

Be $\quad \mathrm{Tr}$

$\mathrm{Hi} \quad \mathrm{Te}$

$\mathrm{Fl}$

04

$\mathrm{Hi} \quad \mathrm{Te}$

$\mathrm{Hi} \quad \mathrm{Te}$

$\mathrm{Hi} \mathrm{Te}$

$\mathrm{FI}$

$\mathrm{FI}$

$\mathrm{Fr}$

$04,06,07$

12

07

$\begin{array}{llll}\mathrm{Hi} & \mathrm{Te} & \mathrm{Fl} & 10 \\ \mathrm{Hi} & \mathrm{Te} & \mathrm{Fl} & 06\end{array}$

$\begin{array}{llll}\text { A } & \text { Te } & \mathrm{Fl} & 11\end{array}$

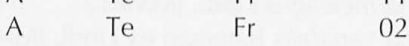

$\mathrm{Hi}$ Te $\mathrm{FI} \quad 07,11,12$

Ar Te

A $\quad$ Te

12

A $\mathrm{Te} \quad \mathrm{Fr}$

$\mathrm{Hi} \mathrm{Te} \quad \mathrm{Fr}$

06

Hi Te FI 04, 07, 08

Be $\quad \mathrm{Te}$

06

$\begin{array}{llll}\text { A } & \text { Te } & \text { Fr } & 06\end{array}$

$\begin{array}{llll}\text { A } & \mathrm{Te} & - & 07\end{array}$

$\begin{array}{llll}\mathrm{Ar} & \mathrm{Te} & - & 02\end{array}$

$\begin{array}{llll}\mathrm{Hi} & \mathrm{Te} & \mathrm{Fl} & 06 \\ \mathrm{Be} & \mathrm{Te} & \mathrm{Fl} & 06\end{array}$




\begin{tabular}{lllcr}
\hline \multicolumn{1}{c}{ Especies y sus datos de colecta } & FC & TH & EF & MC \\
\hline Fischeria scandens DC. JMM1860 & Be & Te & Fl, Fr & 07 \\
Gonolobus chiapensis (Brandegee) Woodson JMM782 & $\mathrm{Be}$ & $\mathrm{Te}$ & $\mathrm{Fl}$ & 04 \\
Gonolobus incerianus W.D.Stevens et Montiel CAPB86, ARA275 & $\mathrm{Be}$ & $\mathrm{Te}$ & $\mathrm{Fl}$ & 04,07 \\
Gonolobus sp. CAPB55 & $\mathrm{Be}$ & $\mathrm{Te}$ & $\mathrm{Fr}$ & 07
\end{tabular}

\section{Asteraceae}

Ageratum echinoides (Less.) Hemsl. JMM693

Ageratum houstonianum Mill. JMM550

Ageratum sp. JMM1126

Alloispermum integrifolium (DC.) H.Rob. JMM873

Bidens sp. JMM658

Chaptalia nutans (L.) Pol. JMM952

Conyza floribunda Kunth JMM 900

Conyza sumatrensis (Retz.) E.Walker var. sumatrensis JMM874

Conyza sumatrensis (Retz.) E.Walker var. leiotheca (S.F. Blake)

Pruski et Sancho JMM934

Cosmos crithmifolius Kunth JMM988

Cosmos sp. JMM593, 1083

Critonia morifolia (Mill.) K. et R. JMM799

Dahlia australis var. chiapensis Turner JMM1137

Erechtites valerianifolia (Wolf) DC. JMM544

Galinsoga parviflora Cav. JMM545, 936

Galinsoga sp. JMM607

Gnaphalium attenuatum DC. JMM939

Gnaphalium roseum Kunth FHN112

Lagascea helianthifolia Kunth JMM655

Lasianthaea fruticosa (L.) K.M.Becker JMM530, 610

Melanthera nivea (L.) Small JMM663, 1080

Montanoa sp. JMM1114

Onoseris onoseroides (Kunth) B.L.Rob. FHN110

Parthenium hysterophorus L. JMM981

Perymenium grande Hemsl. JMM679

Senecio cobanensis J.M.Coult. ARA304, 265

Spilanthes ocymifolia (Lam.) A.H.Moore JMM541

Stevia ovata Willd. JMM626, 695

Tagetes filifolia Lagasca JMM531, 568, 1074

$\begin{array}{ll}\mathrm{Hi} & \mathrm{Te} \\ \mathrm{Hi} & \mathrm{Te} \\ \mathrm{Hi} & \mathrm{Te} \\ \mathrm{Hi} & \mathrm{Te} \\ \mathrm{Ar} & \mathrm{Te} \\ \mathrm{Hi} & \mathrm{Te} \\ \mathrm{Hi} & \mathrm{Te} \\ \mathrm{Hi} & \mathrm{Te} \\ \mathrm{Hi} & \mathrm{Te} \\ & \\ \mathrm{Hi} & \mathrm{Te} \\ \mathrm{Hi} & \mathrm{Te} \\ \mathrm{Ar} & \mathrm{Te} \\ \mathrm{Hi} & \mathrm{Ru} \\ \mathrm{Hi} & \mathrm{Te} \\ \mathrm{Hi} & \mathrm{Te} \\ \mathrm{Hi} & \mathrm{Te} \\ \mathrm{Hi} & \mathrm{Te} \\ \mathrm{Hi} & \mathrm{Te} \\ \mathrm{Hi} & \mathrm{Te} \\ \mathrm{Ar} & \mathrm{Te} \\ \mathrm{Hi} & \mathrm{Te} \\ \mathrm{Hi} & \mathrm{Te} \\ \mathrm{Ar} & \mathrm{Te} \\ \mathrm{Hi} & \mathrm{Te} \\ \mathrm{Ar} & \mathrm{Te} \\ \mathrm{A} & \mathrm{Te} \\ \mathrm{Hi} & \mathrm{Te} \\ \mathrm{Hi} & \mathrm{Te} \\ \mathrm{Hi} & \mathrm{Te} \\ & \end{array}$

\section{Fl}

12

Te $\quad \mathrm{Fl} \quad 11$

Te

FI

11

08

06

Te

$\mathrm{FI}$

12

FI

06

06

FI

$\mathrm{Fl}$

06

Te

FI

06

FI

07

FI

08, 11

04

08

11

06

11

06

02

12

10, 11

08,12

08

02

06

12

04

11

11,12

Balanophoraceae

Helosis cayennensis (Sw.) Spreng. JMM914, CAPB63

$\mathrm{Hi}$

Sa

$08,10,11$

\section{Begoniaceae}

Begonia biserrata Lindl. JMM968

Begonia calderonii Standl. MAPF1795

Begonia corozoensis Ziesenh. CAPB69

Begonia heracleifolia Schltdl. et Cham. JMM1095

Begonia oaxacana A.DC. MAPF1814

Begonia philodendroides Ziesenh. JLC67

Begonia sp. JMM697

$\begin{array}{ll}\mathrm{Hi} & \mathrm{Te} \\ \mathrm{Hi} & \mathrm{Te} \\ \mathrm{Hi} & \mathrm{Te} \\ \mathrm{Hi} & \mathrm{Ru} \\ \mathrm{Hi} & \mathrm{Te} \\ \mathrm{Hi} & \mathrm{Te} \\ \mathrm{Hi} & \mathrm{Ep}\end{array}$

\section{Betulaceae}

Ostrya virginiana (Mill.) K.Koch JLC37

Ostrya virginiana var. guatemalensis (H.J.P.Winkl.) J.F.Macbr.

JMM629, 890, 1119 (Pr)

$\begin{array}{llcc}\text { A } & \text { Te } & \text { Fl } & 07 \\ \text { A } & \text { Te } & \text { Fl, Fr } & 06,08,11\end{array}$

Bignoniaceae

Amphitecna montana L.O.Williams CAPB73

A $\mathrm{Te} \quad \mathrm{Fr}$

07 


\begin{tabular}{|c|c|c|c|c|}
\hline Especies y sus datos de colecta & FC & TH & EF & MC \\
\hline \multicolumn{5}{|l|}{ Boraginaceae } \\
\hline Cordia curassavica (Jacq.) Roem. et Schult. CAPB75 & $\operatorname{Ar}$ & $\mathrm{Te}$ & $\mathrm{FI}$ & 07 \\
\hline Cordia sp. JLC61 & A & $\mathrm{Te}$ & $\mathrm{Fr}$ & 07 \\
\hline Cordia spinescens L. JMM1081, 1110 & $\operatorname{Ar}$ & $\mathrm{Te}$ & $\mathrm{Fl}, \mathrm{Fr}$ & 08 \\
\hline Ehretia tinifolia L. JMM784 & A & Te & $\mathrm{Fl}$ & 04 \\
\hline \multicolumn{5}{|l|}{ Burmanniaceae } \\
\hline Gymnosiphon suaveolens (H.Karst.) Urb. CAPB64 & $\mathrm{Hi}$ & Sa & $\mathrm{Fl}$ & 07 \\
\hline \multicolumn{5}{|l|}{ Cactaceae } \\
\hline Heliocereus elegantissimus Britton et Rose JMM819 & $\mathrm{Hi}$ & Ep & $\mathrm{FI}$ & 04 \\
\hline \multicolumn{5}{|l|}{ Campanulaceae } \\
\hline Lobelia laxiflora Kunth. ARA269, JMM653 & $\mathrm{Hi}$ & Te & $\mathrm{Fl}$ & 04,12 \\
\hline \multicolumn{5}{|l|}{ Capparidaceae } \\
\hline Cleome hemsleyana (Bullock) H.H.Iltis JLC65 & $\mathrm{Hi}$ & $\mathrm{Te}$ & $\mathrm{Fl}$ & 07 \\
\hline Cleome viscosa L. JMM562 & $\mathrm{Hi}$ & Te & $\mathrm{Fl}, \mathrm{Fr}$ & 11 \\
\hline \multicolumn{5}{|l|}{ Caprifoliaceae } \\
\hline Sambucus mexicana C. Presl ex DC. JLC59 & A & $\mathrm{Te}$ & $\mathrm{FI}$ & 07 \\
\hline Viburnum blandum C.V.Morton JMM1082 & $\mathrm{Ar}$ & $\mathrm{Te}$ & $\mathrm{Fl}$ & 08 \\
\hline Viburnum guatemalense Gand. JMM632 & $\operatorname{Ar}$ & $\mathrm{Te}$ & $\mathrm{Fl}, \mathrm{Fr}$ & 11 \\
\hline Viburnum jucundum C.V.Morton JMM597 & $\operatorname{Ar}$ & $\mathrm{Te}$ & $\mathrm{Fl}$ & 11 \\
\hline \multicolumn{5}{|l|}{ Caryophyllaceae } \\
\hline Stellaria ovata Willd. ex Schltdl. ARA294, JMM1030, 1098 & $\mathrm{Be}, \mathrm{Hi}$ & Te, Ru & $\mathrm{FI}$ & $04,07,08$ \\
\hline \multicolumn{5}{|l|}{ Celastraceae } \\
\hline Crossopetalum tonduzii (Loes.) Lundell JMM899, 1035, 1097 & $\mathrm{~A}, \mathrm{Ar}$ & Te & $\mathrm{Fl}$ & $06,07,08$ \\
\hline \multicolumn{5}{|l|}{ Clethraceae } \\
\hline Clethra matudae Lundell JMM882, ARA287 & A & $\mathrm{Te}$ & $\mathrm{FI}$ & 04,06 \\
\hline Clethra mexicana DC. FEPC166 & A & $\mathrm{Te}$ & - & 03 \\
\hline \multicolumn{5}{|l|}{ Clusiaceae } \\
\hline Clusia flava Jacq. JMM917 & A & Te & $\mathrm{Fr}$ & 06 \\
\hline Hypericum formosum Kunth ARA299 & $\mathrm{Hi}$ & $\mathrm{Te}$ & $\mathrm{FI}$ & 04 \\
\hline Tovomitopsis sp. JMM1166, CAPB74 & A & Te & $\mathrm{Fr}$ & 07,08 \\
\hline \multicolumn{5}{|l|}{ Convolvulaceae } \\
\hline Ipomoea hastigera Kunth JMM622 & $\mathrm{Be}$ & Te & $\mathrm{Fl}$ & 11 \\
\hline Ipomoea hederifolia L. FHN122 & $\mathrm{Be}$ & $\mathrm{Te}$ & $\mathrm{Fl}$ & 02 \\
\hline Ipomoea nil (L.) Roth JMM556 & $\mathrm{Be}$ & $\mathrm{Te}$ & $\mathrm{FI}$ & 11 \\
\hline Ipomoea purpurea (L.) Roth CAPB80 & $\mathrm{Be}$ & Te & $\mathrm{Fl}$ & 07 \\
\hline Ipomoea ramosissima (Poir) Choisy JMM535, 631 & $\mathrm{Be}$ & Te & $\mathrm{Fl}$ & 11 \\
\hline Ipomoea silvicola House JMM620, 1001, 1121 & $\mathrm{Be}$ & Te & $\mathrm{FI}$ & $07,08,11$ \\
\hline Ipomoea tiliacea (Willd.) Choisy JMM555, 606, 1076 & $\mathrm{Be}$ & Te & $\mathrm{Fl}$ & 08,11 \\
\hline \multicolumn{5}{|l|}{ Coriariaceae } \\
\hline Coriaria ruscifolia L. JMM700 & $\operatorname{Ar}$ & $\mathrm{Te}$ & $\mathrm{Fl}$ & 12 \\
\hline \multicolumn{5}{|l|}{ Crassulaceae } \\
\hline *Villadia sp. JMM705 & $\mathrm{Hi}$ & $\mathrm{Ru}$ & $\mathrm{FI}$ & 12 \\
\hline \multicolumn{5}{|l|}{ Cucurbitaceae } \\
\hline Melothria pendula L. JMM558, 1067 & $\mathrm{Be}$ & $\mathrm{Te}$ & $\mathrm{FI}$ & 08 \\
\hline
\end{tabular}




\begin{tabular}{l} 
Especies y sus datos de colecta \\
\hline Ericaceae \\
Cavendishia crassifolia (Benth.) Hemsl. JMM814, 1146, ARA305, FEPC152 \\
Vaccinium confertum Kunth JMM703
\end{tabular}

\section{Euphorbiaceae}

Acalypha chiapensis Brandegee JMM1128, CAPB71

Acalypha leptopoda Müll.Arg. JMM630, 891, 1015, ARA278

Acalypha macrostachya Jacq. JLC43

Acalypha unibracteata Müll.Arg. JMM878

Acalypha virgata L. JLC45

Croton draco Schlecht. JMM601, ARA285

Croton guatemalensis Lotsy ARA283

Euphorbia scabrella Boiss. JMM594, 792

Phyllanthus niruri L. JMM667

Phyllanthus grandifolius L. JMM877, 970, 1045

\section{Fabaceae}

Amicia zygomeris DC. JMM588, 1116

Calliandra grandiflora (L'Hér.) Benth. JMM532, 600

Calliandra houstoniana (Miller) Standl. JMM623, 1130, ARA291

Canavalia villosa Benth. JMM567, 573, 965, 1124, ARA279

Centrosema pubescens Benth. JMM875

Chamaecrista rufa (M.Martens et Galeotti) Britton et Rose JMM522, 570, 621

Cojoba haematoloba L. Rico MAPF1860

Crotalaria acapulcensis Hook. et Arn. JMM969, 1115, 1147

Crotalaria bupleurifolia Schltdl. et Cham. JMM590, JLC56

Crotalaria longirostrata Hook. et Arn. JMM1079

Crotalaria sagittalis L. JLC44

Desmodium helleri Peyr. JMM563

Desmodium sp. 1 JMM991

Desmodium sp.2 JMM683

Desmodium sp.3 JMM584

Eriosema diffusum (Kunth) G.Don JMM619, 1093

Erythrina americana Mill. JMM603

Galactia discolor Donn.Sm. JMM884

Indigofera thibaudiana DC. JMM580, 1106

Inga laurina (Sw.) Willd. JLC41

Inga punctata Willd. JMM654

Mimosa albida Humb. et Bonpl. ex Willd. JMM578

Phaseolus coccineus L. JMM937, 1141

Senna sp. JMM659

Tephrosia nicaraguensis Oerst. JMM1018

Teramnus labialis (L.f.) Spreng. JMM1122

Zapoteca aff. portoricensis (Jacq.) H.M.Hern. JMM624, 638

\section{Fagaceae}

Quercus acatenangensis Trel. MAPF1796

Quercus acutifolia Neé JMM902, 922

Quercus candicans Née JMM634

Quercus castanea Née JMM605

Quercus elliptica Née JMM656, 803, 977, ARA290

Quercus magnoliifolia Née JMM604, 892, 976, 1026, MAPF1790

Quercus rugosa Neé JMM1148

Quercus sapotifolia Liebm. MAPF1811

\section{Flacourtiaceae}

Xylosma aff. flexuosa (Kunth) Hemsl. JMM691

FC TH EF $\quad$ MC

$\begin{array}{cccc}\text { Ar, A } & \text { Te } & \text { Fl } & 03,04,08 \\ \text { A } & \text { Te } & \text { Fr } & 12\end{array}$

$\begin{array}{cccc}\mathrm{Hi}, \mathrm{Ar} & \mathrm{Te} & \mathrm{Fl}, \mathrm{Fr} & 07,08 \\ \mathrm{Hi}, \mathrm{Ar} & \mathrm{Te} & \mathrm{FI} & 04,06,07,11 \\ \mathrm{Ar} & \mathrm{Te} & \mathrm{FI} & 07 \\ \mathrm{Hi} & \mathrm{Te} & \mathrm{FI} & 06 \\ \mathrm{Hi} & \mathrm{Te} & \mathrm{FI} & 07 \\ \mathrm{~A} & \mathrm{Te} & \mathrm{Fl}, \mathrm{Fr} & 04,11 \\ \mathrm{~A} & \mathrm{Te} & \mathrm{FI} & 04 \\ \mathrm{Hi} & \mathrm{Te} & \mathrm{FI} & 11 \\ \mathrm{Hi} & \mathrm{Te} & \mathrm{Fl}, \mathrm{Fr} & 12 \\ \mathrm{Ar} & \mathrm{Te} & \mathrm{Fl}, \mathrm{Fr} & 06,07\end{array}$

$\mathrm{H}$

$\mathrm{Ar}$

$\mathrm{Ar}$

Be

Be

$\mathrm{Hi}$

A

$\mathrm{Hi}$

$\mathrm{Hi}$

$\mathrm{Hi}$

$\mathrm{Hi}$

$\mathrm{Hi}$

$\mathrm{Ar}$

$\mathrm{Hi}$

$\mathrm{Hi}$

$\mathrm{Hi}$

A

$\mathrm{Hi}$

Ar

A

A

$\mathrm{Hi}$

Be

Ar

$\mathrm{Hi}$

$\mathrm{Be}$

A

$$
\begin{gathered}
\text { Te } \\
\text { Te } \\
\text { Te, Ru } \\
\text { Te } \\
\text { Te } \\
\text { Te } \\
\text { Te } \\
\text { Te, Ru } \\
\text { Te } \\
\text { Te }
\end{gathered}
$$

FI

$\mathrm{Te}, \mathrm{Ru}$

$\mathrm{Te}$

$\mathrm{Te}$

Te

$\mathrm{Fl}, \mathrm{Fr}$

08,11

$\mathrm{Fl}, \mathrm{Fr}$

$\mathrm{Fl}, \mathrm{Fr}$

$\mathrm{Fl}$

10, 11

04, 08, 11

06, 08

$\mathrm{Fl}, \mathrm{Fr}$

06

$\mathrm{Te}$

Te, Ru

$\mathrm{Te}$

$\mathrm{FI}, \mathrm{Fr}$

10,11

02

Te

$\mathrm{Fl}, \mathrm{Fr}$

FI

$\mathrm{Fl}$

$\mathrm{Fl}$

$\mathrm{Fl}$

$\mathrm{FI}$

FI

$\mathrm{Fl}, \mathrm{Fr}$

$\mathrm{Fr}$

$\mathrm{FI}$

$\mathrm{Fl}, \mathrm{Fr}$

$\mathrm{Fr}$

$\mathrm{Fl}$

$\mathrm{FI}$

$\mathrm{FI}$

$\mathrm{FI}$

$\mathrm{Fl}, \mathrm{Fr}$

$\mathrm{Fl}$

$\mathrm{Fr}$

06, 08

07,11

08

07

11

07

12

11

08, 11

11

06

08,11

07

12

11

06, 08

12
07

08

11

$\begin{array}{ll}\text { A } & \text { Te } \\ \text { A } & \text { Te } \\ \text { A } & \text { Te } \\ \text { A } & \text { Te } \\ \text { A } & \text { Te } \\ \text { A } & \text { Te } \\ \text { A } & \text { Te } \\ \text { A } & \text { Te }\end{array}$

02

06

11

$\begin{array}{cc}\mathrm{Fr} & 11 \\ \mathrm{Fl}, \mathrm{Fr} & 04,06,12\end{array}$

Fr 02, 06, 07, 11

$\mathrm{Fr}$

08

02 


Especies y sus datos de colecta

\section{Gentianaceae}

Centaurium quitense (Kunth) B.L.Rob. JMM657

\section{FC \\ TH}

EF

MC

\section{Gesneriaceae}

Achimenes antirrhina (DC.) Morton JMM 577, 1070

Achimenes candida Lind. JMM1094, 1158, JLC58

Achimenes erecta (Lam.) H.P.Fuchs JMM1060, 1138

Achimenes longiflora DC. JMM515, 521, 1133

Achimenes misera Lindl. JMM518, 1061, 1091

Kohleria deppeana (Schltdl. et Cham.) Fritsch JMM534, 1085

Kohleria rugata (Scheidw.) L.P.Kvist et L.E.Skog JMM1134

Moussonia elegans Decne. JMM576, 1142

Neomortonia nummularia (Hanst.) Wiehler. JMM1012

Niphaea saxifola (Brandegee) D.N.Gibson JMM1104

Solenophora purpusii Brandegee JMM894

\section{Hammamelidaceae}

Liquidambar styraciflua L. JMM1131 (LR)

\section{Hydrophyllaceae}

Wigandia urens (Ruiz et Pav.) Kunth FHN113

$\mathrm{Hi}$

$\mathrm{Te}$

$\mathrm{Fl}$

12

$\begin{array}{ll}\mathrm{Hi} & \mathrm{Te} \\ \mathrm{Hi} & \mathrm{Te}, \mathrm{Ru} \\ \mathrm{Hi} & \mathrm{Ru}, \mathrm{Te} \\ \mathrm{Hi} & \mathrm{Ru}, \mathrm{Te} \\ \mathrm{Hi} & \mathrm{Te}, \mathrm{Ru} \\ \mathrm{Hi} & \mathrm{Te} \\ \mathrm{Hi} & \mathrm{Te} \\ \mathrm{Ar} & \mathrm{Te} \\ \mathrm{Hi} & \mathrm{Ru} \\ \mathrm{Hi} & \mathrm{Ru} \\ \mathrm{Ar} & \mathrm{Te}\end{array}$

\section{Lamiaceae}

Ocimum selloi Benth. JMM995

Salvia cinnabarina M.Martens et Galeotti JMM688

Salvia karwinskii Benth. ARA300

Salvia purpurea Cav. JMM572

Salvia rubiginosa Benth. JMM652

Salvia sp. JMM520

Salvia tonalensis Brandegee JMM587

Scutellaria lundellii Epling JLC63, JMM1139, 1159

\section{Lauraceae}

Cinnamomum sp. JLC74

Nectandra sp. JLC73

Ocotea acuminatissima (Lundell) Rohwer MAPF1807

Ocotea botrantha Rohwer JMM961, CAPB76, JLC7 1

Ocotea chiapensis (Lundell) Standl. et Steyerm. MAPF1819, 1826

Persea americana Mill. JMM999

\section{Loasaceae}

Klaphrothia fasciculata (C.Presl.) Poston JMM585

\section{Loganiaceae}

Spigelia scabra Cham. et Schltdl. JMM517, 551, 789, 926, 1008

\section{Lythraceae}

Cuphea aequipetala Cav. JMM516, 581, 980, 1078

Cuphea appendiculata Benth. JMM524, 895

Cuphea caecilae Koehne JMM685, 1021, 1024

Cuphea sp. JLC66

$\begin{array}{ll}\mathrm{Hi} & \mathrm{Te} \\ \mathrm{Hi} & \mathrm{Te} \\ \mathrm{Hi} & \mathrm{Te} \\ \mathrm{Hi} & \mathrm{Te}\end{array}$

\section{$\mathrm{FI}$}

$\mathrm{FI}$

$\mathrm{Fl}$

FI

\section{7}

07

\section{Malpighiaceae}

Bunchosia lanceolata Turcz. JMM569

Byrsonima crassifolia (L.) Kunth CAPB67

Stigmaphyllon ellipticum (Kunth) A. Juss. JMM525, 571
A $\quad$ Te

Ar $\quad$ Te

Be $\quad \mathrm{Te}$
$06,08,10,11$
06, 10
07,12
07




\begin{tabular}{|c|c|c|c|c|}
\hline Especies y sus datos de colecta & FC & TH & EF & MC \\
\hline \multicolumn{5}{|l|}{ Malvaceae } \\
\hline Anoda cristata (L.) Schltdl. JMM1017, CAPB57 & $\mathrm{Hi}$ & $\mathrm{Te}$ & $\mathrm{FI}$ & 07 \\
\hline Hibiscus uncinellus DC. JMM598, CAPB77 & $\mathrm{Ar}$ & $\mathrm{Te}$ & $\mathrm{FI}$ & 07,11 \\
\hline Malvaviscus arboreus Cav. JMM639, 962, 996, 1113 & $\mathrm{Ar}$ & $\mathrm{Te}$ & $\mathrm{FI}$ & $06,07,08,11$ \\
\hline Malvaviscus arboreus Cav. var. mexicanus Schltdl. MAPF1802 & $\mathrm{Ar}$ & $\mathrm{Te}$ & $\mathrm{Fr}$ & 02 \\
\hline Pavonia rosea Wall. ex Moris JMM787, 929 & $\mathrm{Hi}$ & $\mathrm{Te}$ & $\mathrm{FI}$ & 04,06 \\
\hline Sida acuta Burm.f. JMM540, 1103 & $\mathrm{Hi}$ & $\mathrm{Te}$ & $\mathrm{Fl}, \mathrm{Fr}$ & 08,11 \\
\hline \multicolumn{5}{|l|}{ Melastomataceae } \\
\hline Miconia echinoidea Standl. et Steyerm. JMM989 & $\operatorname{Ar}$ & $\mathrm{Te}$ & $\mathrm{FI}$ & 07 \\
\hline Miconia glaberrima (Schltdl.) Naudin MAPF1792, 1810 & $\operatorname{Ar}$ & $\mathrm{Te}$ & $\mathrm{FI}$ & 02 \\
\hline Miconia guatemalensis Cogn. JMM643, 881, 887, 964, 1117 & $\mathrm{Hi}, \mathrm{Ar}$ & $\mathrm{Te}$ & $\mathrm{Fl}, \mathrm{Fr}$ & $06,08,11$ \\
\hline Monochaetum deppeanum (Schltdl. et Cham.) Naudin ARA293 & $\mathrm{Hi}$ & $\mathrm{Ru}$ & $\mathrm{FI}$ & 04 \\
\hline Monochaetum pulchrum Decne. JMM519, 665 & $\mathrm{Hi}$ & $\mathrm{Te}$ & $\mathrm{Fl}, \mathrm{Fr}$ & 10,12 \\
\hline \multicolumn{5}{|l|}{ Menispermaceae } \\
\hline Cissampelos pareira L. JMM1162 & $\mathrm{Be}$ & $\mathrm{Te}$ & $\mathrm{Fr}$ & 08 \\
\hline Hyperbaena mexicana Miers JLC72 & A & $\mathrm{Te}$ & $\mathrm{FI}$ & 07 \\
\hline \multicolumn{5}{|l|}{ Moraceae } \\
\hline Castilla elastica Sessé ex Cerv. FHN123 & $\mathrm{Hi}$ & $\mathrm{Te}$ & $\mathrm{Fl}$ & 02 \\
\hline Ficus cooki Standl. JMM913 & A & $\mathrm{Te}$ & $\mathrm{Fr}$ & 06 \\
\hline Ficus glabrata Kunth MAPF1820 & A & $\mathrm{Te}$ & - & 02 \\
\hline \multicolumn{5}{|l|}{ Myrsinaceae } \\
\hline Ardisia compressa Kunth. JLC70, ARA266, JMM906 & $\mathrm{A}, \mathrm{Ar}$ & $\mathrm{Te}$ & $\mathrm{Fr}$ & 04,07 \\
\hline Ardisia sp. JMM1011, MAPF1803 & $\mathrm{Ar}$ & $\mathrm{Te}$ & $\mathrm{Fr}$ & 02,07 \\
\hline Myrsine juergensenii (Mez) Ricketson et Pipoly MAPF1797 & A & $\mathrm{Te}$ & - & 02 \\
\hline Parathesis chiapensis Fernald CAPB85, FEPC163, JMM911 & $\mathrm{A}, \mathrm{Ar}$ & $\mathrm{Te}$ & $\mathrm{Fl}, \mathrm{Fr}$ & $03,06,07$ \\
\hline Synardisia venosa (Mast.) Lundell ARA301 & A & Te & $\mathrm{FI}$ & 04 \\
\hline \multicolumn{5}{|l|}{ Myrtaceae } \\
\hline Eugenia acapulcensis Steud. JMM795 & A & $\mathrm{Te}$ & $\mathrm{Fr}$ & 04 \\
\hline Eugenia amatenangensis Lundell JMM946, 955 & $\mathrm{~A}, \mathrm{Ar}$ & $\mathrm{Te}$ & $\mathrm{Fr}$ & 06 \\
\hline Eugenia capuli (Schltdl. et Cham.) Hook. et Arn. JMM791, MAPF1809 & $\mathrm{Ar}, \mathrm{A}$ & $\mathrm{Te}$ & $\mathrm{Fl}$ & 02,04 \\
\hline Myrcianthes sp. JMM701 & A & $\mathrm{Te}$ & $\mathrm{Fl}$ & 12 \\
\hline Psidium guajava L. JMM1129, CAPB62 & $\mathrm{Ar}$ & $\mathrm{Te}$ & $\mathrm{Fr}$ & 07,08 \\
\hline \multicolumn{5}{|l|}{ Ochnaceae } \\
\hline Ouratea nitida (Sw.) Engl. MAPF1821 & $\mathrm{Ar}$ & Te & - & 02 \\
\hline \multicolumn{5}{|l|}{ Onagraceae } \\
\hline Fuchsia encliandra Steud JMM582, 1086 & $\mathrm{Hi}$ & $\mathrm{Te}$ & $\mathrm{Fl}$ & 08,11 \\
\hline Fuchsia sp. JMM611 & $\mathrm{Hi}$ & $\mathrm{Sa}$ & $\mathrm{Fr}$ & 11 \\
\hline Lopezia racemosa Cav. JMM1145 & $\mathrm{Hi}$ & $\mathrm{Ru}$ & $\mathrm{FI}$ & 08 \\
\hline Ludwigia octovalvis (Jacq.) P.H.Raven CAPB66 & $\mathrm{Hi}$ & $\mathrm{Te}$ & $\mathrm{FI}$ & 07 \\
\hline Tibouchina scabriuscula (Schltdl.) Cogn. JMM677 & $\mathrm{Hi}$ & $\mathrm{Ru}$ & $\mathrm{Fl}$ & 12 \\
\hline \multicolumn{5}{|l|}{ Oxalidaceae } \\
\hline Oxalis corniculata L. JMM910 & $\mathrm{Hi}$ & $\mathrm{Te}$ & $\mathrm{Fl}, \mathrm{Fr}$ & 06 \\
\hline \multicolumn{5}{|l|}{ Papaveraceae } \\
\hline Bocconia arborea S.Watson JMM1000 & $\mathrm{Ar}$ & $\mathrm{Te}$ & $\mathrm{Fr}$ & 07 \\
\hline \multicolumn{5}{|l|}{ Passifloraceae } \\
\hline Passiflora biflora Lam. CAPB87 & $\mathrm{Be}$ & $\mathrm{Te}$ & $\mathrm{FI}$ & 07 \\
\hline
\end{tabular}




\begin{tabular}{l} 
Especies y sus datos de colecta \\
\hline Phytolaccaceae \\
Phytolacca icosandra L. JMM546, 953, JLC69, ARA260 \\
Phytolacca purpurascens A.Braun et Bouché JMM1010 \\
Phytolacca sp. JLC77
\end{tabular}

\section{FC}

TH

EF

MC

$\begin{array}{cccc}\mathrm{Hi}, \mathrm{Ar} & \mathrm{Te} & \mathrm{Fl}, \mathrm{fr} & 04,06,07,11 \\ \mathrm{Hi} & \mathrm{Te} & \mathrm{Fl}, \mathrm{Fr} & 07 \\ \mathrm{Hi} & \mathrm{Te} & \mathrm{Fl}, \mathrm{Fr} & 07\end{array}$

\section{Piperaceae}

Peperomia aff. rhexiifolia Moritz ex C.DC. JLC75

Peperomia aggravescens Trel. JMM681

Peperomia asarifolia Schltdl. et Cham. JMM950, 997

Peperomia collocata Trel. ARA303, JMM879, 948, 998

Peperomia esperanzana Trel. JMM811

Peperomia galioides Kunth FEPC136, JMM559

Peperomia macrandra C.DC. JLC54

Peperomia quadrifolia (L.) Kunth FEPC145

Peperomia tetraphylla (G.Forst.) Hook. et Arn. FEPC109, 130

Piper aduncum L. JMM780, 927, 979

Piper holdridgeanum W.C.Burger JMM793

Piper marginatum Jacq. JMM973

Piper sancti-felicis Trel. MAPF1804

Piper schiedeanum Steud. JMM664

Piper umbellatum L. JMM1009

\section{Plantaginaceae}

Plantago major L. JMM788

$\begin{array}{ll}\mathrm{Hi} & \mathrm{Te} \\ \mathrm{Hi} & \mathrm{Ep} \\ \mathrm{Hi} & \mathrm{Ru}, \mathrm{Te} \\ \mathrm{Hi} & \mathrm{Ep} \\ \mathrm{Hi} & \mathrm{Ep} \\ \mathrm{Hi} & \mathrm{Ep} \\ \mathrm{Hi} & \mathrm{Ep} \\ \mathrm{Hi} & \mathrm{Ep} \\ \mathrm{Hi} & \mathrm{Ep} \\ \mathrm{Ar} & \mathrm{Te} \\ \mathrm{Ar} & \mathrm{Te} \\ \mathrm{Ar} & \mathrm{Te} \\ \mathrm{Ar} & \mathrm{Te} \\ \mathrm{Ar} & \mathrm{Te} \\ \mathrm{Ar} & \mathrm{Te}\end{array}$

$\mathrm{FI}$

\section{Platanaceae}

Platanus mexicana Moric. JLC40

\section{Polygalaceae}

Monnina xalapensis Kunth JMM579, 648

Polygala costaricensis Chodat JMM528

Polygala floribunda Benth. JMM669

\section{Pyrolaceae}

Chimaphila maculata (L.) Pursh JMM1044

Monotropa uniflora L. JMM1065

$\mathrm{Hi} \quad \mathrm{Te}$

\section{Ranunculaceae}

Thalictrum galeottii Lecoy. JMM990, 1033

\section{Rhamnaceae}

Rhamnus discolor Lesq. CAPB60

Rhamnus mucronata Schltdl. JMM817

$\begin{array}{llll}\mathrm{A} & \mathrm{Te} & \mathrm{Fr} & 07 \\ \mathrm{Ar} & \mathrm{Te} & \mathrm{Fr} & 04\end{array}$

\section{Rosaceae}

Holodiscus argenteus (L.f.) Maxim. ARA292

Rubus urticifolius Poir. JMM778

Ar Ru

$\mathrm{Fl}$

04

Ar Te

$\mathrm{Fl}, \mathrm{Fr}$

04

\section{Rubiaceae}

Alibertia edulis (Rich.) A.Rich. ex DC. MAPF1817

Arachnothryx laniflora (Benth.) Planch. FEPC162

Arachnothryx pyramidalis (Lundell) Borhidi MAPF1794

Bouvardia longiflora (Cav.) Kunth JMM527, 943, 1028

Crusea coccinea DC. JLC62

Glossostipula aff. strigosa Lorente JMM790

Glossostipula concinna (Standl.) Lorence CAPB81

$\begin{array}{cccc}\text { A } & \text { Te } & \text { Fl } & 02 \\ \text { A } & \text { Te } & - & 03 \\ \mathrm{~A} & \text { Te } & - & 02 \\ \mathrm{Hi} & \mathrm{Te}, \mathrm{Ru} & \mathrm{Fl} & 06,07,10 \\ \mathrm{Hi} & \mathrm{Te} & \mathrm{FI} & 07 \\ \mathrm{~A} & \mathrm{Te} & \mathrm{Fl} & 04 \\ \mathrm{~A} & \mathrm{Te} & - & 07\end{array}$




Especies y sus datos de colecta

Hoffmannia psychotriifolia (Benth.) Griseb. JMM781, 907, 972

Mitracarpus hirtus (L.) DC. JMM538, 938

Palicourea padifolia (Willd. ex Roem. et Schult.) C.M. Taylor et Lorence JMM909, 941, ARA267, JLC32

Psychotria galeottiana (M.Martens) C.M.Taylor et Lorence MAPF1808

Psychotria mexiae Standl. JMM947

Psychotria nervosa Sw. FEPC168, MAPF1789

Rondeletia strigosa (Benth.) Hemsl. JMM678, 1027

$\begin{array}{cccc}\text { FC } & \text { TH } & \text { EF } & \text { MC } \\ \mathrm{Hi} & \mathrm{Te} & \mathrm{FI} & 04,06 \\ \mathrm{Hi} & \mathrm{Te} & \mathrm{Fl} & 06,11 \\ \mathrm{Ar} & \mathrm{Te} & \mathrm{Fl}, \mathrm{Fr} & 04,06,07\end{array}$

\section{Sapindaceae}

Sapindus saponaria L. JMM806

A

$\mathrm{Ar}$

A

$\mathrm{Hi}$

$\mathrm{Te}$

$\mathrm{Fr}$

02

Te

$\mathrm{FI}$

06

Hi $\quad$ Te

$\mathrm{Fl}, \mathrm{Fr}$

02

A

\section{Scrophulariaceae}

Castilleja arvensis Schltdl. et Cham. JMM557

Castilleja chiapensis A.Gray ex Benth. JMM1143, JLC35

Lamourouxia viscosa Kunth JMM640

Russelia multiflora Sims JMM574, 1102

Russelia sarmentosa Jacq. JMM694, 1025

Scoparia dulcis L. JMM706

\section{Simaroubaceae}

Picramnia matudae Lundell MAPF1801

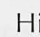

$\mathrm{Hi}$

$\mathrm{Hi}$

$\mathrm{Hi}$

$\mathrm{Hi}$

$\mathrm{Hi}$

Ar

\section{Solanaceae}

Cestrum elegantissimum C.V.Morton JMM596

Cestrum luteo-virescens Francey ARA276

Cestrum nocturnum L. JLC47

Jaltomata procumbens (Cav.) J.L.Gentry JMM553

Lycianthes pilosissimum (M.Martens et Galeotti) Bitter JMM905

Physalis stapelioides (Regel) Bitter JMM609

Solanum ochraceo-ferrugineum (Dunal) Fernald JMM650, 802

Solanum torvum Sw. JMM627, CAPB82

Witheringia meiantha (Donn.Sm.) Hunz. CAPB58, 88

Witheringia solanacea L'Hér. ARA272

\section{Theaceae}

Cleyera theaeoides (Sw.) Choisy MAPF1815, FEPC154

Ternstroemia lineata DC. FEPC149, 171

Ternstroemia tepezapote Schltdl. et Cham. JMM616, 888, 954

\section{Tiliaceae}

Triumfetta speciosa Seem. JMM589, 779, 898, 957, 1107, 1111

\section{Ulmaceae}

Trema micrantha (L.) Blume JMM614, 801, 935, CAPB83

\section{Urticaceae}

Bohemeria sp. JLC64

Phenax hirtus (Sw.) Wedd. JMM583, 586

Pilea aff. auriculata Liebm. JMM1031

Urera alceifolia Gaud. JMM1014

\section{Valerianaceae}

Valeriana sorbifolia Kunth JMM592 


\section{Especies y sus datos de colecta}

\section{Verbenaceae}

Citharexylum caudatum L. ARA280

Citharexylum hexangulare Greenm. FHN124

Hyptis urticoides Kunth JMM613

Lantana achyranthifolia Desf. JMM1034, 1105

Lippia substrigosa Turcz. JMM649, 704, ARA296

Priva lappulacea (L.) Pers. JMM542

Verbena litoralis Kunth JMM608, 928

\section{Viscaceae}

Arceuthobium globosum Hawksw. et Wiens JMM1084

Phoradendron nervosum Oliv. JMM645, JMM958

\section{Vitaceae}

Ampelopsis mexicana Rose JMM959

Vitis tiliifolia Humb. et Bonpl. ex Roem. et Schult. JMM602, CAPB59

\section{Winteraceae}

Drimys granadensis L.f. var. mexicana (DC.) A.C.Sm. FEPC151

\section{FC}

TH

$\begin{array}{cc}\mathrm{A} & \mathrm{T} \\ \mathrm{Ar} & \mathrm{T} \\ \mathrm{Hi} & \mathrm{T} \\ \mathrm{Hi} & \mathrm{T} \\ \mathrm{Ar} & \mathrm{T} \\ \mathrm{Hi} & \mathrm{T} \\ \mathrm{Hi} & \mathrm{T}\end{array}$

EF

MC

Te
Te
Te
Te
Te
Te
Te

$\mathrm{Fr} \quad 04$

$\mathrm{FI} \quad 02$

$\mathrm{FI} \quad 11$

$\mathrm{Fl}, \mathrm{Fr} \quad 07,08$

FI $\quad 04,12$

$\mathrm{FI} \quad 11$

FI 06,11

$\begin{array}{cccc}\mathrm{Hi} & \mathrm{Pa} & \mathrm{Fr} & 08 \\ \mathrm{Hi} & \mathrm{Pa} & \mathrm{Fl}, \mathrm{Fr} & 06,12\end{array}$

Be Te $\quad \mathrm{Fl} \quad 06$

Be $\quad \mathrm{Te} \quad \mathrm{Fl}, \mathrm{Fr} \quad 07,11$

A $\quad$ Te

03 